MATHEMATICS OF COMPUTATION

Volume 76, Number 257, January 2007, Pages 153-177

S $0025-5718(06) 01909-0$

Article electronically published on October 10, 2006

\title{
ANALYSIS OF THE HETEROGENEOUS MULTISCALE METHOD FOR PARABOLIC HOMOGENIZATION PROBLEMS
}

\author{
PINGBING MING AND PINGWEN ZHANG
}

\begin{abstract}
The heterogeneous multiscale method (HMM) is applied to various parabolic problems with multiscale coefficients. These problems can be either linear or nonlinear. Optimal estimates are proved for the error between the HMM solution and the homogenized solution.
\end{abstract}

\section{INTRODUCTION AND MAIN RESULTS}

1.1. Generality. Consider the following parabolic problem:

$$
\left\{\begin{aligned}
\partial_{t} u^{\varepsilon}-\nabla \cdot\left(a^{\varepsilon} \nabla u^{\varepsilon}\right) & =f & & \text { in } D \times(0, T)=: \mathcal{Q}, \\
u^{\varepsilon} & =0 & & \text { on } \partial D \times(0, T), \\
\left.u^{\varepsilon}\right|_{t=0} & =u_{0} . & &
\end{aligned}\right.
$$

Here $\varepsilon$ is a small parameter that signifies the multiscale nature of the problem. We let $D$ be a bounded domain in $\mathbb{R}^{d}$ and $T$ a positive real number. A problem of this type is interesting because of its simplicity and its relevance to several important practical problems, such as the flow in porous media and the mechanical properties of composite materials. In contrast to the elliptic problems there may be oscillations in the temporal direction besides the oscillation in the spatial direction.

On the analytic side, the following fact is known about (1.1). In the sense of parabolic H-convergence (see [25], 8], 12]), introduced with minor modification by Spagnolo and Colombini under the name of G-convergence or PG-convergence (see [11, 22, 23], 24]), for every $f \in L^{2}\left(0, T ; H^{-1}(D)\right)$ and $u_{0} \in L^{2}(D)$, the sequence $\left\{u^{\varepsilon}\right\}$ the solutions of (1.1) satisfies

$$
\begin{aligned}
& u^{\varepsilon} \rightarrow U \quad \text { weakly in } L^{2}\left(0, T ; H_{0}^{1}(D)\right) \text {, } \\
& a^{\varepsilon} \nabla u^{\varepsilon} \rightarrow \mathcal{A} \nabla U \quad \text { weakly in } \quad L^{2}\left(\mathcal{Q} ; \mathbb{R}^{d}\right) \text {, }
\end{aligned}
$$

Received by the editor June 3, 2003 and, in revised form, December 6, 2005.

2000 Mathematics Subject Classification. Primary 65N30, 35K05, 65N15.

Key words and phrases. Heterogeneous multiscale method, parabolic homogenization problems, finite element methods.

The first author was partially supported by the National Natural Science Foundation of China under the grant 10571172 and also supported by the National Basic Research Program under the grant 2005 CB321704.

The second author was partially supported by National Natural Science Foundation of China for Distinguished Young Scholars 10225103 and also supported by the National Basic Research Program under the grant 2005 CB321704.

(C)2006 American Mathematical Society Reverts to public domain 28 years from publication 
where $U$ is the unique solution of the problem

$$
\left\{\begin{aligned}
\partial_{t} U-\nabla \cdot(\mathcal{A} \nabla U) & =f & & \text { in } \mathcal{Q}, \\
U & =0 & & \text { on } \partial D \times(0, T], \\
\left.U\right|_{t=0} & =u_{0} . & &
\end{aligned}\right.
$$

In general, there are no explicit formulas for the effective matrix $\mathcal{A}$.

Classical numerical methods for this problem are designed to resolve the full details of the fine scale problem (1.1) and without taking into account the special features of the coefficient matrix $a^{\varepsilon}$. In contrast, the modern multiscale methods are designed specifically for retrieving partial information about $u^{\varepsilon}$ with sublinear cost [16], i.e., the total cost grows sublinearly with the cost of solving the full fine scale problem. To this end, the methods have to take full advantage of the special features of the problem such as scale separation and self-similarity of the solution. One cannot hope to get an algorithm with sublinear cost for a fully general problem.

The heterogeneous multiscale method introduced in [15] is a general methodology for designing a sublinear algorithm by exploiting the scale separation and other special features of the problem. HMM consists of two ingredients: an overall macroscopic scheme for macrovariables on a macrogrid and estimating the missing macroscopic data from the microscopic model. The efficiency of HMM lies in the ability to extract the missing macroscale data from microscale models with minimum cost, by exploiting scale separation.

For (1.1), the macroscopic solver is chosen to be the standard piecewise linear finite element method 10 over a macroscopic triangulation $\mathcal{T}_{H}$ with mesh size $H$ as the spatial solver, and the backward Euler scheme as the temporal discretization. Many other conventional discretization methods could be proper candidates as the macroscopic solver. For example, the finite difference method and the discontinuous Galerkin method have been employed as the macroscopic solver in [1] and 9], respectively.

We formulate our method as follows. For $1 \leq k \leq n$, let $t_{k}=k \Delta t$ with $\Delta t=T / n$. Let $U_{H}^{0}=Q_{H} u_{0}$ with $Q_{H}$ the $L^{2}$ projection operator from $H_{0}^{1}(D)$ to $X_{H}$, where $X_{H}$ is the macroscopic finite element space. Let $U_{H}^{k} \in X_{H}$ be the solution of the problem

$$
\left(\bar{\partial} U_{H}^{k}, V\right)+A_{H}\left(t_{k} ; U_{H}^{k}, V\right)=\left(f^{k}, V\right) \quad \text { for all } V \in X_{H},
$$

where $\bar{\partial} U_{H}^{k}=\left(U_{H}^{k}-U_{H}^{k-1}\right) / \Delta t$ and $f^{k}=\Delta t^{-1} \int_{t_{k}}^{t_{k}+\Delta t} f(x, s) \mathrm{d} s$.

It remains to estimate the stiffness matrix, which amounts to evaluating the effective bilinear form $A_{H}\left(t_{n} ; V, W\right)$ for any $V, W \in X_{H}$. We write $A_{H}$ as

$$
\begin{aligned}
A_{H}\left(t_{n} ; V, W\right) & =\int_{D} \nabla W \cdot \mathcal{A}_{H}\left(x, t_{n}\right) \nabla V \mathrm{~d} x=\sum_{K \in \mathcal{T}_{H}} \int_{K} \nabla W \cdot \mathcal{A}_{H}\left(x, t_{n}\right) \nabla V \mathrm{~d} x \\
& \simeq \sum_{K \in \mathcal{T}_{H}}|K| \nabla W \cdot \mathcal{A}_{H}\left(x_{K}, t_{n}\right) \nabla V,
\end{aligned}
$$

where $x_{K}$ is the barycenter of $K$. We approximate $\mathcal{A}_{H}\left(x_{K}, t_{n}\right)$ by solving the Cauchy-Dirichlet problem:

$$
\left\{\begin{array}{rlrl}
\partial_{t} v^{\varepsilon}-\nabla \cdot\left(a^{\varepsilon} \nabla v^{\varepsilon}\right) & =0 & & \text { in } \quad\left(x_{K}+I_{\delta}\right) \times\left(t_{n}, t_{n}+\tau_{n}\right), \\
v^{\varepsilon} & =V \\
\left.v^{\varepsilon}\right|_{t=t_{n}} & =V . & & \text { on } \quad \partial I_{\delta} \times\left(t_{n}, t_{n}+\tau_{n}\right),
\end{array}\right.
$$


We then let

$$
\nabla W \cdot \mathcal{A}_{H}\left(x_{K}, t_{n}\right) \nabla V \simeq \frac{1}{\tau_{n}\left|I_{\delta}\right|} \int_{t_{n}}^{t_{n}+\tau_{n}} \int_{I_{\delta}} \nabla w^{\varepsilon} \cdot a^{\varepsilon} \nabla v^{\varepsilon} \mathrm{d} x \mathrm{~d} t,
$$

where $\tau_{n}$ denotes the microsimulation time that evolves in $n$th macrotime step, and $I_{\delta}=\delta Y$ with the unit cell $Y:=(-1 / 2,1 / 2)^{d}$. For simplicity, we denote $I_{\delta}:=x_{K}+I_{\delta}, T_{n}:=\left(t_{n}, t_{n}+\tau_{n}\right)$, and the cylinder $Q_{n}:=I_{\delta} \times T_{n}$. We thus rewrite $A_{H}$ as

$$
A_{H}\left(t_{n} ; V, W\right):=\sum_{K \in \mathcal{T}_{H}} \frac{|K|}{\left|Q_{n}\right|} \int_{Q_{n}} \nabla w^{\varepsilon} \cdot a^{\varepsilon} \nabla v^{\varepsilon} \mathrm{d} x \mathrm{~d} t .
$$

In (1.4), we use the Dirichlet boundary condition and the Cauchy initial condition. One may also use other boundary conditions and initial conditions. For example, we may use the Neumann or periodic boundary condition and the periodic initial condition. In the case when $a^{\varepsilon}=a(x, x / \varepsilon, t)$ and $a(x, y, t)$ is periodic in $y$, one can take $I_{\delta}$ to be $x_{K}+\varepsilon Y$ and impose the boundary/initial conditions, as $v^{\varepsilon}-V$ is periodic on the boundary of the cylinder $\left(x_{K}+\varepsilon Y\right) \times\left(t_{n}, t_{n}+\varepsilon^{2}\right)$.

So far, the algorithm is quite general. The saving compared with solving the full fine scale problem comes from the fact that we may choose $I_{\delta}$ and $\left\{\tau_{k}\right\}$ much smaller than $K$ and $\Delta t$, respectively. The size of the microcell $I_{\delta}$ and the microsimulation time $\left\{\tau_{k}\right\}$ are mainly determined by the accuracy, the cost, and the microstructure of $a^{\varepsilon}$. The main purpose of the error analysis presented below is to help to assess the performance of the method and give guidance for the designing of the methods, namely, how we choose $\delta$ and $\left\{\tau_{k}\right\}$, or types of cell problems.

Since HMM is based on standard macroscale numerical methods and uses the microscale model only as a supplement, it is possible to analyze its stability and accuracy properties using the traditional framework of numerical analysis. This has already been illustrated in [14, 15, 17, and will be further elaborated in the present paper. Roughly speaking, we will show that HMM is stable whenever the macroscopic solver is stable. The overall error between the HMM solution and the homogenized solution is controlled by the accuracy of the macroscopic solver, and the consistency error emanates from the estimate of the macroscopic data from the microscopic model, which will be denoted by $e(\mathrm{HMM})$. Next we estimate $e(\mathrm{HMM})$ for two cases. One is $a^{\varepsilon}=a(x, x / \varepsilon, t)$ with $a(x, y, t)$ periodic in $y$, and the other is $a^{\varepsilon}=a\left(x, x / \varepsilon, t, t / \varepsilon^{2}\right)$ with $a(x, y, t, s)$ periodic in $y$ and $s$.

We will always assume that $a^{\varepsilon}(x, t)$ is symmetric and uniformly elliptic:

$$
\lambda I \leq a^{\varepsilon} \leq \Lambda I
$$

for some $\lambda, \Lambda>0$. We will use $|\cdot|$ to denote the abstract value of a scalar quantity and the volume of a set.

Throughout this paper, the generic constant $C$ is assumed to be independent of the microscale $\varepsilon$, the mesh size $H$, the time step $\Delta t$, the cell size $\delta$, and the microsimulation time $\left\{\tau_{k}\right\}_{k=1}^{n}$. We use the summation convention.

1.2. Main results. Define

$$
e(\mathrm{HMM})=\max _{1 \leq k \leq n} e_{k}(\mathrm{HMM})
$$


with

$$
e_{k}(\mathrm{HMM})=\max _{K \in \mathcal{T}_{H}}\left\|\left(\mathcal{A}-\mathcal{A}_{H}\right)\left(x_{K}, t_{k}\right)\right\|,
$$

where $\|\cdot\|$ denotes the Euclidean norm.

Our main results for the linear problem are as follows.

Theorem 1.1. Let $U$ and $U_{H}^{n}$ be the solutions of (1.2) and (1.3), respectively. If $U$ is sufficiently smooth, then there exists a constant $C$ that is independent of $\varepsilon, \delta,\left\{\tau_{k}\right\}_{k=1}^{n}, H, \Delta t$, such that

$$
\begin{aligned}
\left\|U_{H}^{n}-U\left(x, t_{n}\right)\right\|_{0}+\left\|U_{H}^{n}-U\left(x, t_{n}\right)\right\| & \leq C\left(\Delta t+H^{2}+e(\mathrm{HMM})\right), \\
\left\|U_{H}^{n}-U\left(x, t_{n}\right)\right\|_{1} & \leq C\left(\Delta t+H+e(\mathrm{HMM}) \Delta t^{-1 / 2}\right),
\end{aligned}
$$

where $\||\cdot|\|$ is the weighted space-time $H^{1}$ norm that is defined for every $V=\left\{V^{k}\right\}_{k=1}^{n}$ with $V^{k} \in X$ for $k=1, \ldots, n$ as

$$
\|V\|:=\left(\sum_{k=1}^{n} \Delta t\left\|\nabla V^{k}\right\|_{0}^{2}\right)^{1 / 2} .
$$

At this stage, no assumption on the form of $a^{\varepsilon}$ is necessary. For $U_{H}^{n}$ to converge to $U\left(x, t_{n}\right)$, i.e., $e(\mathrm{HMM}) \rightarrow 0$. $U$ must be chosen as the solution of the homogenized equation, which we now assume exists. To obtain a qualitative estimate for $e(\mathrm{HMM})$, we must make more assumptions on $a^{\varepsilon}$.

We estimate $e(\mathrm{HMM})$ for two special cases that depend on the estimate of the homogenized problem (1.1) presented in the Appendix. The extension to other cases 2, 28 is beyond this paper, since it depends heavily on the qualitative estimates of the corresponding homogenization problem that presently seems missing.

Theorem 1.2. For $a^{\varepsilon}=a(x, x / \varepsilon, t)$ with $a(x, y, t)$ periodic in $y$ with period $Y$, and the cell problem (1.4) is solved with Dirichlet boundary condition and Cauchy initial condition, we have

$$
e(\mathrm{HMM}) \leq C\left[\delta+\frac{\varepsilon}{\delta}+\max _{1 \leq k \leq n}\left(\tau_{k}+\frac{\varepsilon^{2}}{\tau_{k}}\right)\right] .
$$

Another important case for which the estimate of $e(\mathrm{HMM})$ can be obtained is the so-called self-similar case, i.e., $a^{\varepsilon}=a\left(x, x / \varepsilon, t, t / \varepsilon^{2}\right)$. In this case, we have

Theorem 1.3. For $a^{\varepsilon}=a\left(x, x / \varepsilon, t, t / \varepsilon^{2}\right)$ with $a(x, y, t, s)$ periodic in $y$ and $s$ with period $Y$ and 1, respectively, and the cell problem (1.4) solved with the Dirichlet boundary condition and the Cauchy initial condition, we have

$$
e(\mathrm{HMM}) \leq C\left[\delta+\left(\frac{\varepsilon}{\delta}\right)^{1 / 2}+\max _{1 \leq k \leq n}\left(\tau_{k}+\frac{\varepsilon}{\tau_{k}^{1 / 2}}\right)\right] .
$$

Similar results with some modification hold for the nonlinear problems. The details are given in $\$ 4$

1.3. Parameter choices. In this part, we analyze the sources of each term that appears in the upper bound of $e(\mathrm{HMM})$. It is clear that the term $\varepsilon / \delta$ comes from the boundary condition, while the term $\varepsilon^{2} / \tau_{k}$ comes from the initial condition. It is clear to see the corresponding terms vanishes if we let $\delta / \varepsilon, \tau_{k} / \varepsilon^{2} \in \mathbb{N}$, and $v^{\varepsilon}-V$ be periodic on $\partial Q_{n}$. 
For $a^{\varepsilon}=a(x, x / \varepsilon, t)$, we may choose $\delta=M_{1} \varepsilon \simeq \varepsilon^{1 / 2}$ and $\tau_{k}=M_{2} \varepsilon^{2} \simeq \varepsilon$ for $k=1, \ldots, n$. With such a choice of parameters, we get

$$
e(\mathrm{HMM}) \leq C \varepsilon^{1 / 2} \text {. }
$$

For $a^{\varepsilon}=a\left(x, x / \varepsilon, t, t / \varepsilon^{2}\right)$, we may choose $\delta=M_{1} \varepsilon \simeq \varepsilon^{1 / 3}$ and $\tau_{k}=M_{2} \varepsilon^{2} \simeq \varepsilon^{2 / 3}$ for $k=1, \ldots, n$. With such a choice of parameters, we have the overall estimate for $e(\mathrm{HMM})$ as

$$
e(\mathrm{HMM}) \leq C \varepsilon^{1 / 3}
$$

Actually, a formal asymptotical expansion suggests that there is no oscillation in the temporal direction when $a^{\varepsilon}=a(x, x / \varepsilon, t)$. Therefore, we may replace (1.4) by an elliptic cell problem:

$$
\left\{\begin{aligned}
-\nabla \cdot\left(a^{\varepsilon}\left(\cdot, t_{n}\right) \nabla v^{\varepsilon}\right) & =0 & & \text { in } \quad I_{\delta} \\
v^{\varepsilon} & =V & & \text { on } \quad \partial I_{\delta} .
\end{aligned}\right.
$$

Define $w^{\varepsilon}$ in the same way, and $\mathcal{A}_{H}$ is defined as

$$
\nabla W \cdot \mathcal{A}_{H}\left(x_{K}, t_{n}\right) \nabla V=\frac{1}{\left|I_{\delta}\right|} \int_{I_{\delta}} \nabla w^{\varepsilon} \cdot a^{\varepsilon}\left(\cdot, t_{n}\right) \nabla v^{\varepsilon} \mathrm{d} x .
$$

Corollary 1.4. For $a^{\varepsilon}=a(x, x / \varepsilon, t)$ with $a(x, y, t)$ periodic in $y$ with period $Y$, if we use the cell problem (1.13), then

$$
e(\mathrm{HMM}) \leq C\left(\delta+\frac{\varepsilon}{\delta}\right)
$$

The proof of (1.14) is essentially the same as the elliptic case as we have done in [17. Actually, it may also follow the proof of Theorem 1.2 literally; we omit the proof.

\section{AnAlysis of THE Method}

2.1. Preliminaries and notation. We introduce some notation. Denote by $L^{2}(D), H^{m}(D)$ and $H_{0}^{m}(D), m \in \mathbb{Z}$, the usual Lebesgue space and Sobolev spaces. $(\cdot, \cdot)_{D}$ and $\|\cdot\|_{m, D}$ will be denoted as the $L^{2}$ inner-product and norms, respectively, and the subscript will be omitted if no confusion can occur. $f_{D} u \mathrm{~d} x$ is defined as the mean value of $u$ over $D$. For any Banach space $U$ with norm $\|\cdot\|_{U}$, the space $L^{2}(0, T ; U)$ consists of all measurable functions $u:[0, T] \rightarrow U$ with

$$
\|u\|_{L^{2}(0, T ; U)}:=\left(\int_{0}^{T}\|u(t)\|_{U}^{2} \mathrm{~d} t\right)^{1 / 2} .
$$

The space $H^{m}(0, T ; U)$ comprises of all functions $d^{k} u / d t^{k} \in L^{2}(0, T ; U)$ for $0 \leq$ $k \leq m$, which is equipped with the norm

$$
\|u\|_{H^{m}(0, T ; U)}:=\left(\int_{0}^{T} \sum_{0 \leq k \leq m}\left\|d^{k} u / d t^{k}\right\|_{U}^{2} \mathrm{~d} t\right)^{1 / 2} .
$$

The space $\mathcal{C}([0, T] ; U)$ comprises all continuous functions $u:[0, T] \rightarrow U$ with

$$
\|u\|_{\mathcal{C}([0, T] ; U)}=\max _{0 \leq t \leq T}\|u(t)\|_{U}
$$

For vectors $\boldsymbol{x}=\left(x_{1}, x_{2}\right)$ and $\boldsymbol{y}=\left(y_{1}, y_{2}\right) \in \mathbb{R}^{2}, \boldsymbol{x} \otimes \boldsymbol{y}$ is a $2 \times 2$ matrix with elements $(\boldsymbol{x} \otimes \boldsymbol{y})_{i j}:=x_{i} y_{j}$. A matrix product is defined by $A: B=\operatorname{tr}\left(A^{T} B\right)$, where $\operatorname{tr}(A)$ is the trace of a $2 \times 2$ matrix $A$. 
The following simple result underlines the stability of HMM for problem (1.1). A similar one for the elliptic problem can be found in [17, Lemma 1.9].

Lemma 2.1. Given a domain $\Omega \in \mathbb{R}^{d}, T>0$ and a linear function $V$, let $\varphi$ be the solution of

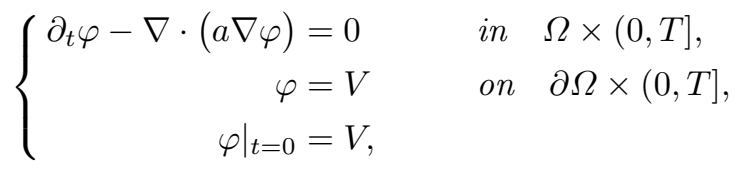

where $a=\left(a_{i j}\right)$ satisfies

$$
\lambda I \leq a \leq \Lambda I \quad \text { a.e. }(x, t) \in \Omega \times(0, T] .
$$

Then for any $t>0$, we have

$$
\|\nabla V\|_{0, \Omega} \leq\|\nabla \varphi(x, t)\|_{0, \Omega} \quad \text { and } \quad\left(\int_{0}^{t} \int_{\Omega} \nabla \varphi \cdot a \nabla \varphi\right)^{1 / 2} \leq\left(\int_{0}^{t} \int_{\Omega} \nabla V \cdot a \nabla V\right)^{1 / 2} .
$$

Proof. Note that $\varphi=V$ on the boundary of $\Omega$, using the fact that $\nabla V$ is a constant in $\Omega$, and integration by parts leads to

$$
\int_{\Omega} \nabla(\varphi-V)(x, t) \nabla V(x) \mathrm{d} x=0 \quad \text { for any } t>0,
$$

which implies

$$
\int_{\Omega}|\nabla \varphi(x, t)|^{2} \mathrm{~d} x=\int_{\Omega}|\nabla V(x)|^{2} \mathrm{~d} x+\int_{\Omega}|\nabla(\varphi-V)(x, t)|^{2} \mathrm{~d} x .
$$

This gives the first result of (2.2). Multiplying the first equation of (2.1) by $\varphi-V$ and integrating by parts, we obtain

$$
\begin{aligned}
\frac{1}{2} \int_{\Omega}|\varphi(x, t)-V|^{2} \mathrm{~d} x & +\int_{0}^{t} \int_{\Omega} \nabla \varphi(x, s) \cdot a(x, s) \nabla \varphi(x, s) \mathrm{d} x \mathrm{~d} s \\
& =\int_{0}^{t} \int_{\Omega} \nabla V(x) \cdot a(x, s) \nabla \varphi(x, s) \mathrm{d} x \mathrm{~d} s .
\end{aligned}
$$

By the Cauchy-Schwartz inequality,

$$
\begin{aligned}
\int_{\Omega} \nabla V(x) \cdot a(x, s) \nabla \varphi(x, s) \mathrm{d} x \mathrm{~d} s \leq & \left(\int_{0}^{t} \int_{\Omega} \nabla \varphi(x, s) \cdot a(x, s) \nabla \varphi(x, s) \mathrm{d} x \mathrm{~d} s\right)^{1 / 2} \\
& \times\left(\int_{0}^{t} \int_{\Omega} \nabla V(x) \cdot a(x, s) \nabla V(x) \mathrm{d} x \mathrm{~d} s\right)^{1 / 2} .
\end{aligned}
$$

A combination of the above two gives the second part of (2.2).

Remark 2.2. For this result, the coefficient $a=\left(a_{i j}\right)$ may depend on the solution, i.e., (2.1) may be nonlinear. 
2.2. Generality. Using (2.2) with $\Omega=I_{\delta}$, for any $V \in X_{H}$ and $1 \leq k \leq n$, we have

$$
\begin{aligned}
A_{H}\left(t_{k} ; V, V\right) & =\sum_{K \in \mathcal{T}_{H}}|K| f_{Q_{k}} \nabla v^{\varepsilon} \cdot a^{\varepsilon} \nabla v^{\varepsilon} \geq \lambda \sum_{K \in \mathcal{T}_{H}}|K| f_{Q_{k}}\left|\nabla v^{\varepsilon}\right|^{2} \\
& \geq \lambda \sum_{K \in \mathcal{T}_{H}}|K| f_{Q_{k}}|\nabla V|^{2}=\lambda \sum_{K \in \mathcal{T}_{H}} \int_{K}|\nabla V|^{2} \\
& =\lambda\|\nabla V\|_{0}^{2} .
\end{aligned}
$$

Similarly, we get

$$
\begin{aligned}
A_{H}\left(t_{k} ; V, W\right) & \leq \sum_{K \in \mathcal{T}_{H}}|K|\left(f_{\mathcal{Q}_{k}} \nabla v^{\varepsilon} \cdot a^{\varepsilon} \nabla v^{\varepsilon}\right)^{1 / 2}\left(f_{\mathcal{Q}_{k}} \nabla w^{\varepsilon} \cdot a^{\varepsilon} \nabla w^{\varepsilon}\right)^{1 / 2} \\
& \leq \sum_{K \in \mathcal{T}_{H}}|K|\left(f_{\mathcal{Q}_{k}} \nabla V \cdot a^{\varepsilon} \nabla V\right)^{1 / 2}\left(f_{\mathcal{Q}_{k}} \nabla W \cdot a^{\varepsilon} \nabla W\right)^{1 / 2} \\
& \leq \Lambda \sum_{K \in \mathcal{T}_{H}}|K||\nabla V||\nabla W|=\Lambda \sum_{K \in \mathcal{T}_{H}}\left(\int_{K}|\nabla V|^{2}\right)^{1 / 2}\left(\int_{K}|\nabla W|^{2}\right)^{1 / 2} \\
& \leq \Lambda\|\nabla V\|_{0}\|\nabla W\|_{0} .
\end{aligned}
$$

The stability of the method is included in the following lemma. The proof is standard by (2.4) and (2.5); we refer to [26] for details.

Lemma 2.3. There exists a constant $C$ such that

$$
\begin{aligned}
\left\|U_{H}^{n}\right\|_{0}+\left\|U_{H}^{n}\right\| & \leq C\left(\left\|u_{0}\right\|_{0}+\left(\sum_{k=1}^{n} \Delta t\left\|f^{k}\right\|_{-1, h}^{2}\right)^{1 / 2}\right), \\
\left\|\nabla U_{H}^{n}\right\|_{0} & \leq C\left(\left\|u_{0}\right\|_{1}+\left(\sum_{k=1}^{n}\left\|f^{k}\right\|_{-1, h}^{2}\right)^{1 / 2}\right)
\end{aligned}
$$

where $\|\cdot\|_{-1, h}$ is defined for any $G \in L^{2}(D)$ as

$$
\|G\|_{-1, h}=\sup _{V \in X_{H}} \frac{(G, V)}{\|\nabla V\|_{0}} .
$$

To prove Theorem 1.1, we define an auxiliary function $\widetilde{U}_{H}^{n} \in X_{H}$ as follows. Let $\widetilde{U}_{H}^{0}=Q_{H} u_{0}$, and for $1 \leq k \leq n, \widetilde{U}_{H}^{k} \in X_{H}$ satisfies

$$
\left(\bar{\partial} \widetilde{U}_{H}^{k}, V\right)+A\left(t_{k} ; \widetilde{U}_{H}^{k}, V\right)=\left(f^{k}, V\right) \quad \text { for all } V \in X_{H},
$$

where $A$ is defined as $A\left(t_{k} ; V, W\right)=\sum_{K \in \mathcal{T}_{H}}|K| \nabla W \cdot \mathcal{A}\left(x_{K}, t_{k}\right) \nabla V$ for all $V, W \in$ $X_{H}$.

The error estimate for the above problem is well known [26]:

$$
\left\|\widetilde{U}_{H}^{n}-U\left(x, t_{n}\right)\right\|_{0}+\left\|\widetilde{U}_{H}^{n}-U\left(x, t_{n}\right)\right\| \leq C\left(\Delta t+H^{2}\right), \quad\left\|\widetilde{U}_{H}^{n}-U\left(x, t_{n}\right)\right\|_{1} \leq C(\Delta t+H) .
$$

Proof of Theorem 1.1, For $1 \leq k \leq n$, define $E^{k}:=U_{H}^{k}-\widetilde{U}_{H}^{k}$. For any $V \in X_{H}$, it is clear that

$$
\left(\bar{\partial} E^{k}, V\right)+A\left(t_{k} ; E^{k}, V\right)=\left(F^{k}, V\right),
$$

where $\left(F^{k}, V\right):=A\left(t_{k} ; U_{H}^{k}, V\right)-A_{H}\left(t_{k} ; U_{H}^{k}, V\right)$. By definition,

$$
\left\|F^{k}\right\|_{-1, h} \leq e_{k}(\mathrm{HMM})\left\|\nabla U_{H}^{k}\right\|_{0} .
$$


By (2.6) we have, since $E^{0}=0$,

$$
\left\|E^{n}\right\|_{0}+\left\|\left|E ^ { n } \left\|\left|\leq C e(\mathrm{HMM})\left\|U_{H}^{n}\right\|\right| \leq C e(\mathrm{HMM}) .\right.\right.\right.
$$

Combining the above inequality and the first part of (2.9), we obtain (1.7).

Repeating the above steps, using (2.7) and (2.6), we obtain

$$
\left\|\nabla E^{n}\right\|_{0} \leq C e(\mathrm{HMM}) \Delta t^{-1 / 2}\left\|U_{H}^{n}\right\| \leq C e(\mathrm{HMM}) \Delta t^{-1 / 2} .
$$

The estimate (1.8) follows from the above estimate and the second part of (2.9)).

Remark 2.4. Noting that $E^{n} \in X_{H}$ for any $n$, and using (2.11) and the inverse estimate [10], we get

$$
\left\|E^{n}\right\|_{1} \leq(C / H)\left\|E^{n}\right\|_{0} \leq C e(\mathrm{HMM}) / H,
$$

which together with the second part of (2.9) leads to

$$
\left\|U_{H}^{n}-U\left(x, t_{n}\right)\right\|_{1} \leq C(H+\Delta t+e(\mathrm{HMM}) / H) .
$$

\section{Estimating $e(\mathrm{HMM})$}

In this section, we estimate $e(\mathrm{HMM})$ for two cases: one is $a^{\varepsilon}=a(x, x / \varepsilon, t)$ and the other is $a^{\varepsilon}=a\left(x, x / \varepsilon, t, t / \varepsilon^{2}\right)$. In both cases, the cell problem (1.4) is solved with the Dirichlet boundary condition and the Cauchy initial condition. We will use $a_{K, n}^{\varepsilon}=a\left(x_{K}, x / \varepsilon, t_{n}\right)$ or $a_{K, n}^{\varepsilon}=a\left(x_{K}, x / \varepsilon, t_{n}, t / \varepsilon^{2}\right)$ and $\chi_{K, n}=\chi\left(x_{K}, x / \varepsilon, t_{n}\right)$ or $\chi_{K, n}=\chi\left(x_{K}, x / \varepsilon, t_{n}, t / \varepsilon^{2}\right)$ for simplicity, where $\chi$ is the solution of certain cell problems (cf. (3.4) and (3.15)).

Estimating $e(\mathrm{HMM})$ consists of two steps. First, we estimate $\|\widetilde{\mathcal{A}}-\mathcal{A}\|$. The auxiliary operator $\widetilde{\mathcal{A}}$ is defined by

$$
\nabla W \cdot \widetilde{\mathcal{A}}\left(x_{K}, t_{n}\right) \nabla V=f_{Q_{n}} \nabla \widehat{W}^{\varepsilon} \cdot a_{K, n}^{\varepsilon} \nabla \widehat{V}^{\varepsilon} \quad \text { for any } W, V \in X_{H},
$$

where

$$
\widehat{V}^{\varepsilon}=V+\varepsilon \boldsymbol{\chi}_{K, n} \cdot \nabla V \quad \text { and } \quad \widehat{W}^{\varepsilon}=W+\varepsilon \boldsymbol{\chi}_{K, n} \cdot \nabla W .
$$

Next we estimate $\left\|\widetilde{\mathcal{A}}-\mathcal{A}_{H}\right\|$. This is achieved by

$$
\begin{aligned}
\nabla W \cdot & \left(\widetilde{\mathcal{A}}-\mathcal{A}_{H}\right)\left(x_{K}, t_{n}\right) \nabla V \\
= & f_{Q_{n}}\left[\nabla \widehat{W}^{\varepsilon} \cdot a_{K, n}^{\varepsilon} \nabla\left(\widehat{V}^{\varepsilon}-v^{\varepsilon}\right)+\nabla \widehat{V}^{\varepsilon} \cdot a_{K, n}^{\varepsilon} \nabla\left(\widehat{W}^{\varepsilon}-w^{\varepsilon}\right)\right] \\
& \quad-f_{Q_{n}}\left[\nabla w^{\varepsilon} \cdot\left(a^{\varepsilon}-a_{K, n}^{\varepsilon}\right) \nabla v^{\varepsilon}+\nabla\left(w^{\varepsilon}-\widehat{W}^{\varepsilon}\right) \cdot a_{K, n}^{\varepsilon} \nabla\left(v^{\varepsilon}-\widehat{V}^{\varepsilon}\right)\right] .
\end{aligned}
$$

Finally, estimating $e(\mathrm{HMM})$ follows from the triangle inequality.

3.1. Estimating $e(\mathbf{H M M})$ for the case when $a^{\varepsilon}=a(x, x / \varepsilon, t)$. Denote by $\hat{v}^{\varepsilon}$ the solution of (1.4) with $a^{\varepsilon}$ replaced by $a_{K, n}^{\varepsilon}$. By a standard a priori estimate and (2.2), we have

$$
\left\|\nabla\left(v^{\varepsilon}-\hat{v}^{\varepsilon}\right)\right\|_{L^{2}\left(Q_{n}\right)} \leq C\left(\delta+\tau_{n}\right)\left\|\nabla v^{\varepsilon}\right\|_{L^{2}\left(\varrho_{n}\right)} \leq C\left(\delta+\tau_{n}\right)\|\nabla V\|_{L^{2}\left(\varrho_{n}\right)} .
$$

For $j=1, \ldots, d, \chi=\left\{\chi^{j}\right\}_{j=1}^{d}$ is periodic in $y$ with period $Y$ and satisfies

$$
\frac{\partial}{\partial y_{i}}\left(a_{i k} \frac{\partial \chi^{j}}{\partial y_{k}}\right)(x, y, t)=-\left(\frac{\partial}{\partial y_{i}} a_{i j}\right)(x, y, t) \quad \text { in } Y, \quad \int_{Y} \chi^{j}(x, y, t) \mathrm{d} y=0
$$


This problem is solvable, and there exists a constant $C$ such that for $j=1, \ldots, d$,

$$
\left|\nabla_{y} \chi^{j}(x, y, t)\right| \leq C \quad \text { for all }(x, t) \in \mathcal{Q} \text { and } y \in Y \text {. }
$$

The effective matrix is given by

$$
\mathcal{A}_{i j}(x, t)=f_{Y}\left(a_{i j}+a_{i k} \frac{\partial \chi^{j}}{\partial y_{k}}\right)(x, y, t) \mathrm{d} y \quad i, j=1, \ldots, d .
$$

A straightforward calculation gives

$$
\nabla \cdot\left(a_{K, n}^{\varepsilon} \nabla \widehat{V}^{\varepsilon}\right)=0 \quad \text { and } \quad \nabla \cdot\left(a_{K, n}^{\varepsilon} \nabla \widehat{W}^{\varepsilon}\right)=0 .
$$

Define $\theta^{\varepsilon}=\hat{v}^{\varepsilon}-\widehat{V}^{\varepsilon}$, which obviously satisfies

$$
\left\{\begin{aligned}
\partial_{t} \theta^{\varepsilon}-\nabla \cdot\left(a_{K, n}^{\varepsilon} \nabla \theta^{\varepsilon}\right) & =0 & & \text { in } \quad Q_{n}, \\
\theta^{\varepsilon} & =-\varepsilon \boldsymbol{\chi}_{K, n} \cdot \nabla V & & \text { on } \quad \partial I_{\delta} \times T_{n}, \\
\left.\theta^{\varepsilon}\right|_{t=t_{n}} & =-\varepsilon \boldsymbol{\chi}_{K, n} \cdot \nabla V . & &
\end{aligned}\right.
$$

Lemma 3.1. Let $\theta^{\varepsilon}$ be solution of (3.8). There exists a constant independent of $\varepsilon, \delta$, and $\tau_{n}$ such that

$$
\left\|\nabla \theta^{\varepsilon}\right\|_{L^{2}\left(Q_{n}\right)} \leq C\left(\frac{\varepsilon}{\tau_{n}^{1 / 2}}+\left(\frac{\varepsilon}{\delta}\right)^{1 / 2}\right)\|\nabla V\|_{L^{2}\left(Q_{n}\right)} .
$$

Proof. Multiplying both sides of (3.8) $)_{1}$ by $\theta_{1}^{\varepsilon}:=\theta^{\varepsilon}+\left(\widehat{V}^{\varepsilon}-V\right)\left(1-\rho^{\varepsilon}\right)$ and integrating over $I_{\delta}$, we obtain

$$
\frac{1}{2} \frac{\partial}{\partial t} \int_{I_{\delta}}\left|\theta_{1}^{\varepsilon}\right|^{2}+\int_{I_{\delta}} \nabla \theta_{1}^{\varepsilon} \cdot a_{K, n}^{\varepsilon} \nabla \theta_{1}^{\varepsilon}=\int_{I_{\delta}} \nabla\left(\theta_{1}^{\varepsilon}-\theta^{\varepsilon}\right) \cdot a_{K, n}^{\varepsilon} \nabla \theta_{1}^{\varepsilon},
$$

where the cut-off function $\rho^{\varepsilon} \in C_{0}^{\infty}\left(I_{\delta}\right),\left|\nabla \rho^{\varepsilon}\right| \leq C / \varepsilon$, and

$$
\rho^{\varepsilon}= \begin{cases}1 & \text { if } \operatorname{dist}\left(x, \partial I_{\delta}\right) \geq 2 \varepsilon \\ 0 & \text { if } \operatorname{dist}\left(x, \partial I_{\delta}\right) \leq \varepsilon\end{cases}
$$

It is clear to see that

$$
\left|\int_{I_{\delta}} \nabla\left(\theta_{1}^{\varepsilon}-\theta^{\varepsilon}\right) \cdot a_{K, n}^{\varepsilon} \nabla \theta_{1}^{\varepsilon}\right| \leq\left(\int_{I_{\delta}} \nabla\left(\theta_{1}^{\varepsilon}-\theta^{\varepsilon}\right) \cdot a_{K, n}^{\varepsilon} \nabla\left(\theta_{1}^{\varepsilon}-\theta^{\varepsilon}\right)\right)^{1 / 2}\left(\int_{I_{\delta}} \nabla \theta_{1}^{\varepsilon} \cdot a_{K, n}^{\varepsilon} \nabla \theta_{1}^{\varepsilon}\right)^{1 / 2} \text {. }
$$

Substituting the above inequality into (3.10), we obtain

$$
\frac{\partial}{\partial t} \int_{I_{\delta}}\left|\theta_{1}^{\varepsilon}\right|^{2}+\int_{I_{\delta}} \nabla \theta_{1}^{\varepsilon} \cdot a_{K, n}^{\varepsilon} \nabla \theta_{1}^{\varepsilon} \leq \int_{I_{\delta}} \nabla\left(\theta_{1}^{\varepsilon}-\theta^{\varepsilon}\right) \cdot a_{K, n}^{\varepsilon} \nabla\left(\theta_{1}^{\varepsilon}-\theta^{\varepsilon}\right) .
$$

Integrating the above inequality over $T_{n}$, we get

$$
\lambda\left\|\nabla \theta_{1}^{\varepsilon}\right\|_{L^{2}\left(Q_{n}\right)}^{2} \leq\left\|\theta_{1}^{\varepsilon}\left(x, t_{n}\right)\right\|_{L^{2}\left(I_{\delta}\right)}^{2}+\Lambda\left\|\nabla\left(\theta_{1}^{\varepsilon}-\theta^{\varepsilon}\right)\right\|_{L^{2}\left(\Omega_{n}\right)}^{2},
$$

which implies

$$
\left\|\nabla \theta^{\varepsilon}\right\|_{L^{2}\left(Q_{n}\right)} \leq \lambda^{-1 / 2}\left\|\theta_{1}^{\varepsilon}\left(x, t_{n}\right)\right\|_{L^{2}\left(I_{\delta}\right)}+\left(1+(\Lambda / \lambda)^{1 / 2}\right)\left\|\nabla\left(\theta_{1}^{\varepsilon}-\theta^{\varepsilon}\right)\right\|_{L^{2}\left(Q_{n}\right)} .
$$

A direct calculation gives

$$
\begin{aligned}
\left\|\nabla\left(\theta_{1}^{\varepsilon}-\theta^{\varepsilon}\right)\right\|_{L^{2}\left(Q_{n}\right)} & \leq C\left(\frac{\varepsilon}{\delta}\right)^{1 / 2}\|\nabla V\|_{L^{2}\left(Q_{n}\right)}, \\
\left\|\theta_{1}^{\varepsilon}\left(x, t_{n}\right)\right\|_{L^{2}\left(I_{\delta}\right)} & =\varepsilon\left\|\rho^{\varepsilon}\left(\widehat{V}^{\varepsilon}-V\right)\right\|_{L^{2}\left(I_{\delta}\right)} \leq C \varepsilon\|\nabla V\|_{L^{2}\left(I_{\delta}\right)} .
\end{aligned}
$$

A combination of the above three inequalities leads to (3.9).

Next lemma concerns estimating $\|\widetilde{\mathcal{A}}-\mathcal{A}\|$. 
Lemma 3.2. There exists a constant $C$ such that

$$
\left\|(\widetilde{\mathcal{A}}-\mathcal{A})\left(x_{K}, t_{n}\right)\right\| \leq C \frac{\varepsilon}{\delta} .
$$

Proof. Denote by $I_{\kappa \varepsilon}=\kappa Y$, where $\kappa$ is the integer part of $\delta / \varepsilon$, i.e., $\kappa=\lfloor\delta / \varepsilon\rfloor$, integrating by parts and using (3.7), we get

$$
f_{I_{\kappa \varepsilon}} \nabla\left(\widehat{W}^{\varepsilon}-W\right) \cdot a_{K, n}^{\varepsilon} \nabla \widehat{V}^{\varepsilon}=0
$$

Using the expression of $\widehat{V}^{\varepsilon}$ and (3.6), we obtain

$$
f_{I_{\kappa \varepsilon}} \nabla W \cdot a_{K, n}^{\varepsilon} \nabla \widehat{V}^{\varepsilon}=\nabla W \cdot \mathcal{A}\left(x_{K}, t_{n}\right) \nabla V .
$$

It follows from the above two equations that

$$
f_{I_{\kappa \varepsilon}} \nabla \widehat{W}^{\varepsilon} \cdot a_{K, n}^{\varepsilon} \nabla \widehat{V}^{\varepsilon}=\nabla W \cdot \mathcal{A}\left(x_{K}, t_{n}\right) \nabla V .
$$

Since $\widehat{V}^{\varepsilon}, \widehat{W}^{\varepsilon}$ and $a_{K, n}^{\varepsilon}$ are independent of $t$, we write $\widetilde{\mathcal{A}}$ as

$$
\nabla W \cdot \widetilde{\mathcal{A}}\left(x_{K}, t_{n}\right) \nabla V=f_{I_{\delta}} \nabla \widehat{W}^{\varepsilon} \cdot a_{K, n}^{\varepsilon} \nabla \widehat{V}^{\varepsilon} \quad \text { for any } W, V \in X_{H} .
$$

It follows from the above equation and (3.5) that

$$
\begin{aligned}
\mid \nabla W & \cdot(\mathcal{A}-\widetilde{\mathcal{A}})\left(x_{K}, t_{n}\right) \nabla V \mid \\
& \leq\left(1-\frac{\left|I_{\kappa \varepsilon}\right|}{\left|I_{\delta}\right|}\right) f_{I_{\kappa \varepsilon}}\left|\nabla W \cdot a_{K, n}^{\varepsilon} \nabla \widehat{V}^{\varepsilon}\right|+\left|I_{\delta}\right|^{-1} \int_{I_{\delta} \backslash I_{\kappa \varepsilon}}\left|\nabla W \cdot a_{K, n}^{\varepsilon} \nabla \widehat{V}^{\varepsilon}\right| \\
& \leq C \frac{\varepsilon}{\delta}|\nabla W||\nabla V|,
\end{aligned}
$$

which in turn implies (3.11).

Proof of (1.9). Using the first part of (3.7) and noting that

$$
\left[\widehat{W}^{\varepsilon} \rho^{\varepsilon}-w^{\varepsilon}+W\left(1-\rho^{\varepsilon}\right)\right](x, t)=0
$$

for $(x, t) \in \partial I_{\delta} \times T_{n}$, integrating by parts, we have

$$
f_{Q_{n}} \nabla \widehat{V}^{\varepsilon} \cdot a_{K, n}^{\varepsilon} \nabla\left(\widehat{W}^{\varepsilon} \rho^{\varepsilon}-w^{\varepsilon}+W\left(1-\rho^{\varepsilon}\right)\right)=0 .
$$

Therefore, we get

$$
\begin{aligned}
f_{Q_{n}} \nabla \widehat{V}^{\varepsilon} \cdot a_{K, n}^{\varepsilon} \nabla\left(\widehat{W}^{\varepsilon}-w^{\varepsilon}\right) & =f_{Q_{n}} \nabla \widehat{V}^{\varepsilon} \cdot a_{K, n}^{\varepsilon} \nabla\left[\left(\widehat{W}^{\varepsilon}-W\right)\left(1-\rho^{\varepsilon}\right)\right] \\
& =f_{I_{\delta}} \nabla \widehat{V}^{\varepsilon} \cdot a_{K, n}^{\varepsilon} \nabla\left[\left(\widehat{W}^{\varepsilon}-W\right)\left(1-\rho^{\varepsilon}\right)\right] .
\end{aligned}
$$

Symmetrically, using the second part of (3.7), we have

$$
f_{Q_{n}} \nabla \widehat{W}^{\varepsilon} \cdot a_{K, n}^{\varepsilon} \nabla\left(\widehat{V}^{\varepsilon}-v^{\varepsilon}\right)=f_{I_{\delta}} \nabla \widehat{W}^{\varepsilon} \cdot a_{K, n}^{\varepsilon} \nabla\left[\left(\widehat{V}^{\varepsilon}-V\right)\left(1-\rho^{\varepsilon}\right)\right] .
$$


Using the above two identities, we rewrite (3.2) as

$$
\begin{aligned}
& \nabla W \cdot\left(\widetilde{\mathcal{A}}-\mathcal{A}_{H}\right)\left(x_{K}, t_{n}\right) \nabla V \\
&=f_{I_{\delta}}\left[\nabla \widehat{W}^{\varepsilon} \cdot a_{K, n}^{\varepsilon} \nabla\left[\left(\widehat{V}^{\varepsilon}-V\right)\left(1-\rho^{\varepsilon}\right)\right]\right. \\
&\left.+\nabla \widehat{V}^{\varepsilon} \cdot a_{K, n}^{\varepsilon} \nabla\left[\left(\widehat{W}^{\varepsilon}-W\right)\left(1-\rho^{\varepsilon}\right)\right]\right] \\
&-f_{\mathcal{Q}_{n}}\left[\nabla w^{\varepsilon} \cdot\left(a^{\varepsilon}-a_{K, n}^{\varepsilon}\right) \nabla v^{\varepsilon}\right. \\
&\left.+\nabla\left(w^{\varepsilon}-\widehat{W}^{\varepsilon}\right) \cdot a_{K, n}^{\varepsilon} \nabla\left(v^{\varepsilon}-\widehat{V}^{\varepsilon}\right)\right] \\
&=: I_{1}+I_{2} .
\end{aligned}
$$

A direct calculation gives

$$
\left|I_{1}\right| \leq C \frac{\varepsilon}{\delta}|\nabla W||\nabla V|
$$

It follows from (3.3) and (3.9) that

$$
\begin{aligned}
\left\|\nabla\left(v^{\varepsilon}-\widehat{V}^{\varepsilon}\right)\right\|_{L^{2}\left(Q_{n}\right)} & \leq\left\|\nabla\left(v^{\varepsilon}-\hat{v}^{\varepsilon}\right)\right\|_{L^{2}\left(Q_{n}\right)}+\left\|\nabla \theta^{\varepsilon}\right\|_{L^{2}\left(Q_{n}\right)} \\
& \leq C\left(\delta+\tau_{n}+\left(\frac{\varepsilon}{\delta}\right)^{1 / 2}+\frac{\varepsilon}{\tau_{n}^{1 / 2}}\right)\|\nabla V\|_{L^{2}\left(Q_{n}\right)} .
\end{aligned}
$$

Similarly, we have

$$
\left\|\nabla\left(w^{\varepsilon}-\widehat{W}^{\varepsilon}\right)\right\|_{L^{2}\left(Q_{n}\right)} \leq C\left(\delta+\tau_{n}+\left(\frac{\varepsilon}{\delta}\right)^{1 / 2}+\frac{\varepsilon}{\tau_{n}^{1 / 2}}\right)\|\nabla W\|_{L^{2}\left(Q_{n}\right)} .
$$

Using the above two inequalities, we obtain

$$
\begin{aligned}
\left|I_{2}\right| \leq & C \frac{\delta+\tau_{n}}{\left|Q_{n}\right|}\left\|\nabla w^{\varepsilon}\right\|_{L^{2}\left(Q_{n}\right)}\left\|\nabla v^{\varepsilon}\right\|_{L^{2}\left(Q_{n}\right)} \\
& +\frac{\Lambda}{\left|Q_{n}\right|}\left\|\nabla\left(w^{\varepsilon}-\widehat{W}^{\varepsilon}\right)\right\|\left\|_{L^{2}\left(Q_{n}\right)}\right\| \nabla\left(v^{\varepsilon}-\widehat{V}^{\varepsilon}\right) \|_{L^{2}\left(Q_{n}\right)} \\
\leq & C\left|Q_{n}\right|^{-1}\left(\delta+\tau_{n}+\frac{\varepsilon}{\delta}+\frac{\varepsilon^{2}}{\tau_{n}}\right)\|\nabla W\|_{L^{2}\left(Q_{n}\right)}\|\nabla V\|_{L^{2}\left(Q_{n}\right)} \\
= & C\left(\delta+\tau_{n}+\frac{\varepsilon}{\delta}+\frac{\varepsilon^{2}}{\tau_{n}}\right)|\nabla W||\nabla V| .
\end{aligned}
$$

Summing up the estimates for $I_{1}$ and $I_{2}$, we obtain

$$
\left\|\left(\widetilde{\mathcal{A}}-\mathcal{A}_{H}\right)\left(x_{K}, t_{n}\right)\right\| \leq C\left(\delta+\tau_{n}+\frac{\varepsilon}{\delta}+\frac{\varepsilon^{2}}{\tau_{n}}\right),
$$

which together with (3.11) gives (1.9).

3.2. Estimating $e(\mathbf{H M M})$ for the case when $a^{\varepsilon}=a\left(x, x / \varepsilon, t, t / \varepsilon^{2}\right)$. Next we estimate $e(\mathrm{HMM})$ for the case $a^{\varepsilon}=a\left(x, x / \varepsilon, t, t / \varepsilon^{2}\right)$ when $a(x, y, t, s)$ is periodic in $y$ and $s$ with period $Y$ and 1, respectively. We assume that (1.4) is solved with the Dirichlet boundary condition and the Cauchy initial condition. For $j=1, \ldots, d$, $\chi(x, y, t, s)=\left\{\chi^{j}\right\}_{j=1}^{d}$ is periodic in $y$ and $s$ with periods $Y$ and 1 , respectively, and satisfies

$$
\partial_{s} \chi^{j}-\partial_{y_{i}}\left(a_{i k} \frac{\partial \chi^{j}}{\partial y_{k}}\right)(x, y, t, s)=\left(\partial_{y_{i}} a_{i j}\right)(x, y, t, s) \quad \text { and } \int_{0}^{1} \int_{Y} \chi^{j}(x, y, t, s) \mathrm{d} y \mathrm{~d} s=0 .
$$


The existence of $\chi^{j}$ is obvious since

$$
\int_{0}^{1} \int_{Y}\left(\partial_{y_{i}} a_{i j}\right)(x, y, t, s) \mathrm{d} y \mathrm{~d} s=0 .
$$

By [20, there exists a constant $C$ such that for $j=1, \ldots, d$,

$$
\left|\chi^{j}(x, y, s, t)\right|+\left|\nabla_{y} \chi^{j}(x, y, s, t)\right| \leq C \quad \text { for all }(x, t) \in \mathcal{Q}, y \in Y \text { and } s \in(0,1) .
$$

Denote by $\hat{v}^{\varepsilon}$ the solution of (1.4) with $a^{\varepsilon}$ replaced by $a_{K, n}^{\varepsilon}$. Using the standard a priori estimate and Lemma 2.1. we have

$$
\left\|\nabla\left(v^{\varepsilon}-\hat{v}^{\varepsilon}\right)\right\|_{L^{2}\left(Q_{n}\right)} \leq C\left(\delta+\tau_{n}\right)\|\nabla V\|_{L^{2}\left(Q_{n}\right)} .
$$

It is easy to verify that

$$
\partial_{t} \widehat{V}^{\varepsilon}-\nabla \cdot\left(a_{K, n}^{\varepsilon} \nabla \widehat{V}^{\varepsilon}\right)=0 \quad \text { and } \quad \partial_{t} \widehat{W}^{\varepsilon}-\nabla \cdot\left(a_{K, n}^{\varepsilon} \nabla \widehat{W}^{\varepsilon}\right)=0,
$$

and

$$
\left\{\begin{aligned}
\partial_{t} \theta^{\varepsilon}-\nabla \cdot\left(a_{K, n}^{\varepsilon} \nabla \theta^{\varepsilon}\right) & =0 & & \text { in } \quad \mathcal{Q}_{n}, \\
\theta^{\varepsilon} & =-\varepsilon \boldsymbol{\chi}_{K, n} \cdot \nabla V & & \text { on } \quad \partial I_{\delta} \times T_{n}, \\
\left.\theta^{\varepsilon}\right|_{t=t_{n}} & =-\left.\varepsilon\left(\boldsymbol{\chi}_{K, n} \cdot \nabla V\right)\right|_{t=t_{n}} . & &
\end{aligned}\right.
$$

For the correction $\theta^{\varepsilon}$, we have the following estimate (cf. (3.9)).

Lemma 3.3. There exists a constant $C$ independent of $\varepsilon, \delta$, and $\tau_{n}$ such that

$$
\left\|\nabla \theta^{\varepsilon}\right\|_{L^{2}\left(Q_{n}\right)} \leq C\left(\left(\frac{\varepsilon}{\delta}\right)^{1 / 2}+\frac{\varepsilon}{\tau_{n}^{1 / 2}}\right)\|\nabla V\|_{L^{2}\left(Q_{n}\right)} .
$$

The proof of (3.20) is essentially the same as Lemma 3.1. The difference lies in the second term in the right-hand side of the equation below.

Proof. Multiplying both sides of $(\underline{3.19})_{1}$ by $\theta_{1}^{\varepsilon}:=\theta^{\varepsilon}+\left(\widehat{V}^{\varepsilon}-V\right)\left(1-\rho^{\varepsilon}\right)$ and integrating by parts, we get

$$
\frac{1}{2} \frac{\partial}{\partial t} \int_{I_{\delta}}\left|\theta_{1}^{\varepsilon}\right|^{2}+\int_{I_{\delta}} \nabla \theta_{1}^{\varepsilon} \cdot a_{K, n}^{\varepsilon} \nabla \theta_{1}^{\varepsilon}=\int_{I_{\delta}} \nabla \theta_{1}^{\varepsilon} \cdot a^{\varepsilon} \nabla\left(\theta_{1}^{\varepsilon}-\theta^{\varepsilon}\right)+\frac{1}{2} \int_{I_{\delta}} \theta_{1}^{\varepsilon} \partial_{t}\left(\theta_{1}^{\varepsilon}-\theta^{\varepsilon}\right)
$$

It follows from (3.15) that

$$
\begin{aligned}
\int_{I_{\delta}} \theta_{1}^{\varepsilon} \partial_{t}\left(\theta_{1}^{\varepsilon}-\theta^{\varepsilon}\right) & =\varepsilon^{-1} \int_{I_{\delta}} \partial_{s} \chi_{K, n} \cdot \nabla V\left(1-\rho^{\varepsilon}\right) \theta_{1}^{\varepsilon} \\
& =\varepsilon^{-1} \int_{I_{\delta}} \nabla_{y} \cdot\left(a_{K, n}^{\varepsilon}\left(I+\nabla_{y} \chi_{K, n}\right)\right) \nabla V\left(1-\rho^{\varepsilon}\right) \theta_{1}^{\varepsilon} \\
& =\int_{I_{\delta}} \nabla \cdot\left(a_{K, n}^{\varepsilon}\left(I+\nabla_{y} \chi_{K, n}\right)\right) \nabla V\left(1-\rho^{\varepsilon}\right) \theta_{1}^{\varepsilon} .
\end{aligned}
$$

Integrating by parts, we obtain

$$
\begin{aligned}
\int_{I_{\delta}} \theta_{1}^{\varepsilon} \partial_{t}\left(\theta_{1}^{\varepsilon}-\theta^{\varepsilon}\right)= & -\int_{I_{\delta}} \nabla\left(\theta_{1}^{\varepsilon}\left(1-\rho^{\varepsilon}\right) \nabla V\right): a_{K, n}^{\varepsilon}\left(I+\nabla_{y} \chi_{K, n}\right) \\
= & -\int_{I_{\delta}}\left(1-\rho^{\varepsilon}\right)\left[\nabla \theta_{1}^{\varepsilon} \otimes \nabla V\right]: a_{K, n}^{\varepsilon}\left(I+\nabla_{y} \chi_{K, n}\right) \\
& +\int_{I_{\delta}} \theta_{1}^{\varepsilon} \nabla \rho^{\varepsilon} \cdot a_{K, n}^{\varepsilon}\left(I+\nabla_{y} \chi_{K, n}\right) \nabla V
\end{aligned}
$$


Using (3.16), we bound the first term in the right-hand side of the above equation as

$$
\begin{aligned}
\mid \int_{I_{\delta}} & \left(1-\rho^{\varepsilon}\right)\left[\nabla \theta_{1}^{\varepsilon} \otimes \nabla V\right]: a_{K, n}^{\varepsilon}\left(I+\nabla_{y} \chi_{K, n}\right) \mid \\
& \leq \Lambda \max _{(x, t) \in Q_{n}}\left\|I+\nabla_{y} \chi_{K, n}\right\|\left\|\nabla \theta_{1}^{\varepsilon}\right\|_{L^{2}\left(I_{\delta}\right)}\|\nabla V\|_{L^{2}\left(I_{\delta} \backslash I_{(\kappa-2) \varepsilon)}\right)} \\
& \leq C\left(\frac{\varepsilon}{\delta}\right)^{1 / 2}\left\|\nabla \theta_{1}^{\varepsilon}\right\|_{L^{2}\left(I_{\delta}\right)}\|\nabla V\|_{L^{2}\left(I_{\delta}\right)} .
\end{aligned}
$$

By maximum principle 20], we have

$$
\max _{(x, t) \in \mathcal{Q}_{n}}\left|\theta^{\varepsilon}(x, t)\right| \leq \varepsilon \max _{(x, t) \in \mathcal{Q}_{n}}\left|\chi_{K, n}(x, t)\right||\nabla V| .
$$

We thus get

$$
\begin{aligned}
\max _{(x, t) \in \mathcal{Q}_{n}}\left|\theta_{1}^{\varepsilon}(x, t)\right| & \leq \max _{(x, t) \in \mathcal{Q}_{n}}\left(\left|\theta^{\varepsilon}(x, t)\right|+\varepsilon\left|\chi_{K, n}(x, t)\right||\nabla V|\right) \\
& \leq 2 \varepsilon \max _{(x, t) \in \mathcal{Q}_{n}}\left|\chi_{K, n}(x, t)\right||\nabla V| .
\end{aligned}
$$

Therefore, we bound the second term in the right-hand side of (3.22) as

$$
\begin{aligned}
& \left|\int_{I_{\delta}} \theta_{1}^{\varepsilon} \nabla \rho^{\varepsilon} \cdot a_{K, n}^{\varepsilon}\left(I+\nabla_{y} \chi_{K, n}\right) \nabla V\right| \\
& \quad \leq 2 \Lambda \max _{(x, t) \in Q_{n}}\left\|I+\nabla_{y} \chi_{K, n}(x, t)\right\| \int_{I_{\delta}}|\nabla V|^{2}\left|\varepsilon \nabla \rho^{\varepsilon}\right| \\
& \quad \leq C \frac{\varepsilon}{\delta}\|\nabla V\|_{L^{2}\left(I_{\delta}\right)}^{2} .
\end{aligned}
$$

Substituting the above two estimates into (3.21), we obtain

$$
\begin{aligned}
& \frac{1}{2} \frac{\partial}{\partial t} \int_{I_{\delta}}\left|\theta_{1}^{\varepsilon}\right|^{2}+\int_{I_{\delta}} \nabla \theta_{1}^{\varepsilon} \cdot a_{K, n}^{\varepsilon} \nabla \theta_{1}^{\varepsilon} \\
& \leq \frac{1}{2} \int_{I_{\delta}} \nabla \theta_{1}^{\varepsilon} \cdot a_{K, n}^{\varepsilon} \nabla \theta_{1}^{\varepsilon}+\int_{I_{\delta}} \nabla\left(\theta^{\varepsilon}-\theta_{1}^{\varepsilon}\right) \cdot a_{K, n}^{\varepsilon} \nabla\left(\theta^{\varepsilon}-\theta_{1}^{\varepsilon}\right) \\
& \quad+C \frac{\varepsilon}{\delta}\|\nabla V\|_{L^{2}\left(I_{\delta}\right)}^{2} .
\end{aligned}
$$

Therefore, integrating the above inequality over $T_{n}$, we obtain

$$
\left\|\nabla \theta_{1}^{\varepsilon}\right\|_{L^{2}\left(\Omega_{n}\right)} \leq C\left(\left\|\theta_{1}^{\varepsilon}\left(x, t_{n}\right)\right\|_{L^{2}\left(I_{\delta}\right)}+\left\|\nabla\left(\theta^{\varepsilon}-\theta_{1}^{\varepsilon}\right)\right\|_{L^{2}\left(\Omega_{n}\right)}+\left(\frac{\varepsilon}{\delta}\right)^{1 / 2}\|\nabla V\|_{L^{2}\left(\Omega_{n}\right)}\right),
$$

which in turn implies

$\left\|\nabla \theta^{\varepsilon}\right\|_{L^{2}\left(\Omega_{n}\right)} \leq C\left(\left\|\theta_{1}^{\varepsilon}\left(x, t_{n}\right)\right\|_{L^{2}\left(I_{\delta}\right)}+C\left\|\nabla\left(\theta^{\varepsilon}-\theta_{1}^{\varepsilon}\right)\right\|_{L^{2}\left(\Omega_{n}\right)}+C\left(\frac{\varepsilon}{\delta}\right)^{1 / 2}\|\nabla V\|_{L^{2}\left(\Omega_{n}\right)}\right)$.

A direct calculation gives

$$
\begin{aligned}
\left\|\theta_{1}^{\varepsilon}\left(x, t_{n}\right)\right\|_{L^{2}\left(I_{\delta}\right)} & \leq C \varepsilon\|\nabla V\|_{L^{2}\left(I_{\delta}\right)}, \\
\left\|\nabla\left(\theta^{\varepsilon}-\theta_{1}^{\varepsilon}\right)\right\|_{L^{2}\left(Q_{n}\right)} & \leq C\left(\frac{\varepsilon}{\delta}\right)^{1 / 2}\|\nabla V\|_{L^{2}\left(\Omega_{n}\right)} .
\end{aligned}
$$

A combination of the above three inequalities leads to (3.20).

Similar to Lemma 3.2, we have 
Lemma 3.4. There exists a constant $C$ such that

$$
\left\|(\mathcal{A}-\widetilde{\mathcal{A}})\left(x_{K}, t_{n}\right)\right\| \leq C\left(\frac{\varepsilon}{\delta}+\frac{\varepsilon^{2}}{\tau_{n}}\right) .
$$

Proof. Let $\ell:=\left\lfloor\tau_{n} / \varepsilon^{2}\right\rfloor$ and $\widetilde{Q}_{n}:=I_{\kappa \varepsilon} \times\left(t_{n}, t_{n}+\ell \varepsilon^{2}\right)$. The key to the proof is the following observation: for any $V, W \in X_{H}$, we have

$$
\nabla W \cdot \mathcal{A}\left(x_{K}, t_{n}\right) \nabla V:=f_{\widetilde{\Omega}_{n}} \nabla \widehat{W}^{\varepsilon} \cdot a_{K, n}^{\varepsilon} \nabla \widehat{V}^{\varepsilon} .
$$

Integration by parts and using the first part of (3.18), we obtain

$$
\begin{aligned}
f_{\widetilde{\Omega}_{n}} & \nabla\left(\widehat{W}^{\varepsilon}-W\right) \cdot a_{K, n}^{\varepsilon} \nabla \widehat{V}^{\varepsilon} \\
& =-f_{\widetilde{\Omega}_{n}}\left(\widehat{W}^{\varepsilon}-W\right) \nabla \cdot\left(a_{K, n}^{\varepsilon} \nabla \widehat{V}^{\varepsilon}\right)=-f_{\widetilde{\Omega}_{n}}\left(\widehat{W}^{\varepsilon}-W\right) \partial_{t} \widehat{V}^{\varepsilon} \\
& =-f_{\widetilde{\Omega}_{n}}\left(\widehat{W}^{\varepsilon}-W\right) \partial_{t}\left(\widehat{V}^{\varepsilon}-V\right) .
\end{aligned}
$$

A direct calculation leads to

$$
f_{\widetilde{Q}_{n}} \nabla W \cdot a_{K, n}^{\varepsilon} \nabla \widehat{V}^{\varepsilon}=\nabla W \cdot \mathcal{A}\left(x_{K}, t_{n}\right) \nabla V .
$$

Adding up the above two equations, we obtain

$$
\nabla W \cdot \mathcal{A}\left(x_{K}, t_{n}\right) \nabla V-f_{\widetilde{Q}_{n}} \nabla \widehat{W}^{\varepsilon} \cdot a_{K, n}^{\varepsilon} \nabla \widehat{V}^{\varepsilon}=f_{\widetilde{Q}_{n}}\left(\widehat{W}^{\varepsilon}-W\right) \partial_{t}\left(\widehat{V}^{\varepsilon}-V\right) .
$$

Exchanging $W$ and $V$ and noting that $a^{\varepsilon}$ and $\mathcal{A}$ are symmetric, we get

$$
\nabla W \cdot \mathcal{A}\left(x_{K}, t_{n}\right) \nabla V-f_{\widetilde{Q}_{n}} \nabla \widehat{W}^{\varepsilon} \cdot a_{K, n}^{\varepsilon} \nabla \widehat{V}^{\varepsilon}=f_{\widetilde{Q}_{n}}\left(\widehat{V}^{\varepsilon}-V\right) \partial_{t}\left(\widehat{W}^{\varepsilon}-W\right) .
$$

Adding up the above two equations and using the explicit expressions of $\widehat{V}^{\varepsilon}$ and $\widehat{W}^{\varepsilon}$, we get

$$
\nabla W \cdot \mathcal{A}\left(x_{K}, t_{n}\right) \nabla V-f_{\widetilde{\Omega}_{n}} \nabla \widehat{W}^{\varepsilon} \cdot a_{K, n}^{\varepsilon} \nabla \widehat{V}^{\varepsilon}=\frac{1}{2} f_{\widetilde{\Omega}_{n}} \partial_{t}\left[\left(\widehat{V}^{\varepsilon}-V\right)\left(\widehat{W}^{\varepsilon}-W\right)\right]=0,
$$

which gives (3.25).

By (3.25), proceeding as in (3.12) and using (3.16), we get (3.24).

Proof of (1.10). It follows from (3.2), (3.17) and Lemma 3.3 that

$$
\begin{aligned}
\left|\nabla W \cdot\left(\widetilde{\mathcal{A}}-\mathcal{A}_{H}\right)\left(x_{K}, t_{n}\right) \nabla V\right| \leq & C\left(\delta+\tau_{n}+\left(\frac{\varepsilon}{\delta}\right)^{1 / 2}+\frac{\varepsilon}{\tau_{n}^{1 / 2}}\right)|\nabla W||\nabla V| \\
& +C \frac{\delta+\tau_{n}}{\left|Q_{n}\right|}\left\|\nabla w^{\varepsilon}\right\|_{L^{2}\left(Q_{n}\right)}\left\|\nabla v^{\varepsilon}\right\|_{L^{2}\left(Q_{n}\right)} \\
& +\frac{\Lambda}{\left|Q_{n}\right|}\left\|\nabla\left(w^{\varepsilon}-\widehat{W}^{\varepsilon}\right)\right\|_{L^{2}\left(Q_{n}\right)}\left\|\nabla\left(v^{\varepsilon}-\widehat{V}^{\varepsilon}\right)\right\|_{L^{2}\left(Q_{n}\right)} \\
\leq & C\left(\delta+\tau_{n}+\left(\frac{\varepsilon}{\delta}\right)^{1 / 2}+\frac{\varepsilon}{\tau_{n}^{1 / 2}}\right)|\nabla W \| \nabla V|,
\end{aligned}
$$

which implies

$$
\left\|\left(\widetilde{\mathcal{A}}-\mathcal{A}_{H}\right)\left(x_{K}, t_{n}\right)\right\| \leq C\left(\delta+\tau_{n}+\left(\frac{\varepsilon}{\delta}\right)^{1 / 2}+\frac{\varepsilon}{\tau_{n}^{1 / 2}}\right) .
$$


This estimate together with (3.24) leads to (1.10).

Remark 3.5. One may wonder whether the estimate (1.10) can be improved to (1.9). This is actually not the case due to (3.26).

\section{NONLINEAR PROBLEM}

We consider the following nonlinear problem

$$
\left\{\begin{aligned}
\partial_{t} u^{\varepsilon}-\nabla \cdot\left(a^{\varepsilon}\left(x, t, u^{\varepsilon}\right) \nabla u^{\varepsilon}\right) & =f & & \text { in } \quad 2, \\
u^{\varepsilon} & =0 & & \text { on } \quad \partial D \times(0, T), \\
\left.u^{\varepsilon}\right|_{t=0} & =u_{0} . & &
\end{aligned}\right.
$$

We assume that $a^{\varepsilon}\left(x, t, u^{\varepsilon}\right)$ satisfies

$$
\lambda|\xi|^{2} \leq a_{i j}^{\varepsilon}(x, t, z) \xi_{i} \xi_{j} \leq \Lambda|\xi|^{2} \quad \text { for all } \xi \in \mathbb{R}^{d} \text { and for all }(x, t) \in \mathcal{Q} \text { and } z \in \mathbb{R}
$$

with $0<\lambda \leq \Lambda$. Moreover, we assume that $a^{\varepsilon}(x, t, z)$ is Lipschitz continuous in $z$ uniformly with respect to $x$ and $t$. The existence of $u^{\varepsilon}$ is classic. A similar problem in the elliptic case has been discussed in [7, and the extension to (4.1) is straightforward. We refer to [19] for more general nonlinear problems. The homogenized problem, if it exists, is of the following form:

$$
\left\{\begin{aligned}
\partial_{t} U-\nabla \cdot(\mathcal{A}(x, t, U) \nabla U) & =f & & \text { in } \quad Q, \\
U & =0 & & \text { on } \quad \partial D \times(0, T), \\
\left.U\right|_{t=0} & =u_{0} . & &
\end{aligned}\right.
$$

To formulate HMM, for any $V \in X_{H}$, define $v^{\varepsilon}$ to be the solution of

$$
\left\{\begin{aligned}
\partial_{t} v^{\varepsilon}-\nabla \cdot\left(a^{\varepsilon}\left(x, t, v^{\varepsilon}\right) \nabla v^{\varepsilon}\right) & =0 & & \text { in } \quad Q_{n}, \\
v^{\varepsilon} & =V & & \text { on } \quad \partial I_{\delta} \times T_{n}, \\
\left.v^{\varepsilon}\right|_{t=t_{n}} & =V . & &
\end{aligned}\right.
$$

We can define $w^{\varepsilon}$ similarly.

For any $V, W \in X_{H}$, we define

$$
\nabla W \cdot \mathcal{A}_{H}\left(x_{K}, t_{n}, V\right) \nabla V:=\int_{Q_{n}} \nabla w^{\varepsilon} \cdot a^{\varepsilon}\left(x, t, v^{\varepsilon}\right) \nabla v^{\varepsilon},
$$

and $A_{H}\left(t_{n} ; V, W\right)=\sum_{K \in \mathcal{T}_{H}}|K| \nabla W \cdot \mathcal{A}_{H}\left(x_{K}, t_{n}, V\right) \nabla V$.

The HMM solution is given by the following problem.

Problem 4.1. Let $U_{H}^{0}=Q_{H} u_{0}$, for $k=1, \ldots, n$, and find $U_{H}^{k} \in X_{H}$ such that

$$
\left(\bar{\partial} U_{H}^{k}, V\right)+A_{H}\left(t_{k} ; U_{H}^{k}, V\right)=\left(f^{k}, V\right) \quad \text { for all } V \in X_{H} .
$$

Remark 4.2. Though we only consider a special nonlinear problem, the algorithm applies to a much general nonlinear problem (cf. [19]) that together with realistic application will be dealt with in a forthcoming paper.

For any $V, W \in X_{H}$, we define

$$
E_{k}(V, W):=\nabla W \cdot\left(\mathcal{A}_{H}-\mathcal{A}\right)\left(x_{K}, t_{k}, V\right) \nabla V
$$

and

$$
e(\mathrm{HMM})=\max _{\substack{K \in \mathcal{T}_{H}, V \in X_{H} \\ 1 \leq k \leq n}} \frac{E_{k}(V, W)}{|\nabla W||\nabla V|} .
$$


Proceeding along the same line of Lemma 2.1, we get the same estimate for $v^{\varepsilon}$. Note that $a^{\varepsilon}$ in the second part of (2.2) depends on the solution $v^{\varepsilon}$. Obviously, for any $V \in X_{H}$, we have

$$
A_{H}\left(t_{k} ; V, V\right) \geq \lambda\|\nabla V\|_{0}^{2} .
$$

By (4.5), it is easy to derive a stability result that is similar to (2.6) and (2.7).

Similar to the second part of (2.2), for any $W \in X_{H}$, we have

$$
\left(\int_{0}^{t} \int_{\Omega} \nabla w^{\varepsilon} \cdot a^{\varepsilon}\left(x, t, w^{\varepsilon}\right) \nabla w^{\varepsilon}\right)^{1 / 2} \leq\left(\int_{0}^{t} \int_{\Omega} \nabla W \cdot a^{\varepsilon}\left(x, t, w^{\varepsilon}\right) \nabla W\right)^{1 / 2} .
$$

Using the above inequality, we get

$$
\begin{aligned}
A_{H}\left(t_{k} ; V, W\right) \leq & \sum_{K \in \mathcal{T}_{H}}|K|\left(\frac{\Lambda}{\lambda}\right)^{1 / 2} \\
& \cdot\left(f_{Q_{k}} \nabla v^{\varepsilon} \cdot a^{\varepsilon}\left(x, t, v^{\varepsilon}\right) \nabla v^{\varepsilon}\right)^{1 / 2}\left(f_{Q_{k}} \nabla w^{\varepsilon} \cdot a^{\varepsilon}\left(x, t, w^{\varepsilon}\right) \nabla w^{\varepsilon}\right)^{1 / 2} \\
\leq & \sum_{K \in \mathcal{T}_{H}}|K|\left(\frac{\Lambda}{\lambda}\right)^{1 / 2}\left(f_{Q_{k}} \nabla V \cdot a^{\varepsilon}\left(x, t, v^{\varepsilon}\right) \nabla V\right)^{1 / 2} \\
& \cdot\left(f_{Q_{k}} \nabla W \cdot a^{\varepsilon}\left(x, t, w^{\varepsilon}\right) \nabla W\right)^{1 / 2} \\
\leq & \Lambda\left(\frac{\Lambda}{\lambda}\right)^{1 / 2} \sum_{K \in \mathcal{T}_{H}}|K||\nabla V||\nabla W| \\
= & \Lambda\left(\frac{\Lambda}{\lambda}\right)^{1 / 2} \sum_{K \in \mathcal{T}_{H}}\left(\int_{K}|\nabla V|^{2}\right)^{1 / 2}\left(\int_{K}|\nabla W|^{2}\right)^{1 / 2} \\
\leq & \Lambda(\Lambda / \lambda)^{1 / 2}\|\nabla V\|_{0}\|\nabla W\|_{0} .
\end{aligned}
$$

The existence of the solution easily follows from the standard approach in [13] by (4.5) and (4.6), while the uniqueness is more involved, which together with the error estimate will be addressed in Theorem 4.3 .

The error estimate for Problem 4.1 is essentially the same as the linear case. Define $\widetilde{U}_{H}^{n}$ as: let $\widetilde{U}_{H}^{0}=Q_{H} u_{0}$, for $k=1, \ldots, n$, and $\widetilde{U}_{H}^{k} \in X_{H}$ satisfies

$$
\left(\bar{\partial} \widetilde{U}_{H}^{k}, V\right)+A\left(t_{k} ; \widetilde{U}_{H}^{k}, V\right)=\left(f^{k}, V\right) \quad \text { for all } V \in X_{H},
$$

where

$$
A\left(t_{k} ; \widetilde{U}_{H}^{k}, V\right)=\sum_{K \in \mathcal{T}_{H}}|K| \nabla V \cdot \mathcal{A}\left(x_{K}, t_{k}, \widetilde{U}_{H}^{k}\right) \nabla \widetilde{U}_{H}^{k}
$$

For simplicity of notation, we associate $A$ with an operator $\hat{\mathcal{A}}$ as

$$
\left(\hat{\mathcal{A}}\left(x, t_{k}, V\right) \nabla V, \nabla W\right)=A\left(t_{k} ; V, W\right) \quad \text { for all } V, W \in X_{H} .
$$

By [7, Theorem 3.1], the effective matrix $\mathcal{A}$ satisfies

$$
\lambda I \leq \mathcal{A} \leq\left(\Lambda^{2} / \lambda\right) I .
$$

Moreover, by [7. Proposition 3.5], $\mathcal{A}(x, t, z)$ (so does $\hat{\mathcal{A}}$ ) is Lipschitz continuous in $z$ uniformly with respect to all $(x, t) \in \mathcal{Q}$, and the Lipschitz constant is denoted by L. By 26],

$$
\left\|\widetilde{U}_{H}^{n}-U\left(x, t_{n}\right)\right\|_{0} \leq C\left(\Delta t+H^{2}\right)
$$


and there exists a constant $K_{1}:=C_{*}\left(\Delta t^{1 / 2}+H+\Delta t / H\right)$ such that

$$
\Delta t^{1 / 2}\left\|\nabla \widetilde{U}_{H}^{n}\right\|_{L^{\infty}} \leq K_{1}
$$

where $C_{*}$ depends on $U$.

Theorem 4.3. Let $U$ and $U_{H}^{n}$ be solutions of (4.2) and (4.4), respectively. Then, under the appropriate regularity assumption on $U$, we have, for small $\Delta t$,

$$
\left\|U_{H}^{n}-U\left(x, t_{n}\right)\right\|_{0} \leq C\left(H^{2}+\Delta t+e(\mathrm{HMM})\right) .
$$

Moreover, for $M=K_{1}+C H^{-1} e(\mathrm{HMM})$ with $C$ a generic constant independent of $\varepsilon, \delta, H, \tau_{n}, X, Z$, and $V$, if $M$ satisfies

$$
L^{2} M^{2}<\lambda
$$

and there exists a constant $\eta(M)$ with $0<\eta(M)<\lambda / 2$ such that

$$
\int_{D}\left|E_{k}(X, V)-E_{k}(Z, V)\right| \mathrm{d} x \leq \eta(M)\|X-Z\|_{1}\|\nabla V\|_{0}
$$

for all $X, Z \in X_{H} \cap W^{1, \infty}(D)$ and $V \in X_{H}$ satisfying $\|X\|_{1, \infty},\|Z\|_{1, \infty} \leq M$, then the HMM solution is locally unique.

Proof. Define $E^{n}=U_{H}^{n}-\widetilde{U}_{H}^{n}$; we have for any $V \in X_{H}$,

$$
\begin{aligned}
\left(\bar{\partial} E^{k}, V\right)+\left(\hat{\mathcal{A}}\left(x, t_{k}, U_{H}^{k}\right) \nabla E^{k}, \nabla V\right)= & \left(A-A_{H}\right)\left(t_{k} ; U_{H}^{k}, V\right) \\
& +\left(\left(\hat{\mathcal{A}}\left(x, t_{k}, \widetilde{U}_{H}^{k}\right)-\hat{\mathcal{A}}\left(x, t_{k}, U_{H}^{k}\right)\right) \nabla \widetilde{U}_{H}^{k}, \nabla V\right) .
\end{aligned}
$$

Taking $V=E^{k}$ in the above equation and using (4.5), we get

$$
\begin{aligned}
\frac{1}{2 \Delta t}\left(\left\|E^{k}\right\|_{0}^{2}-\left\|E^{k-1}\right\|_{0}^{2}\right)+\lambda\left\|\nabla E^{k}\right\|_{0}^{2} \leq & e(\mathrm{HMM})\left\|\nabla U_{H}^{k}\right\|_{0}\left\|\nabla E^{k}\right\|_{0} \\
& +C\left\|\nabla \widetilde{U}_{H}^{k}\right\|_{L^{\infty}}\left\|E^{k}\right\|_{0}\left\|\nabla E^{k}\right\|_{0} .
\end{aligned}
$$

Using (4.8) and a kickback of $\left\|\nabla E^{k}\right\|$, we get

$$
\frac{1}{2 \Delta t}\left(\left\|E^{k}\right\|_{0}^{2}-\left\|E^{k-1}\right\|_{0}^{2}\right)+\frac{\lambda}{2}\left\|\nabla E^{k}\right\|_{0}^{2} \leq\left(e^{2}(\mathrm{HMM}) / \lambda\right)\left\|\nabla U_{H}^{k}\right\|_{0}^{2}+C\left\|E^{k}\right\|_{0}^{2}
$$

There exists a constant $M_{1}$ such that for $\Delta t<M_{1}$, there holds

$$
\left\|E^{k}\right\|_{0}^{2} \leq(1+C \Delta t)\left\|E^{k-1}\right\|_{0}^{2}+C \Delta t e^{2}(\mathrm{HMM})\left\|\nabla U_{H}^{k}\right\|_{0}^{2} .
$$

Hence, by recursive application of the above inequality and noting that $E^{0}=0$, we obtain

$$
\left\|E^{n}\right\|_{0}^{2} \leq C e^{2}(\mathrm{HMM}) \Delta t \sum_{k=1}^{n}(1+C \Delta t)^{n-k}\left\|\nabla U_{H}^{k}\right\|_{0}^{2} \leq C e^{2}(\mathrm{HMM})\left\|U_{H}\right\|^{2} .
$$

This together with (4.7) gives (4.9).

Let $U_{H}^{n}=X$ and $U_{H}^{n}=Z$ be solutions of Problem 4.1 with $U_{H}^{n-1}$ given. Then by substraction, we get for all $V \in X_{H}$,

$$
(X-Z, V)+\Delta t A_{H}\left(t_{n} ; X, V\right)=\Delta t A_{H}\left(t_{n} ; Z, V\right),
$$

which can be rewritten as

$$
\begin{aligned}
(X- & Z, V)+\Delta t\left(\hat{\mathcal{A}}\left(x, t_{n}, X\right) \nabla(X-Z), \nabla V\right) \\
= & \Delta t\left(A_{H}-A\right)\left(t_{n} ; Z, V\right)-\Delta t\left(A_{H}-A\right)\left(t_{n} ; X, V\right) \\
& +\Delta t\left(\left[\hat{\mathcal{A}}\left(x, t_{n}, Z\right)-\hat{\mathcal{A}}\left(x, t_{n}, X\right)\right] \nabla Z, \nabla V\right) .
\end{aligned}
$$


Taking $V=X-Z$ in the above equation and using (4.11), we get

$$
\begin{aligned}
\|X-Z\|_{0}^{2}+\lambda \Delta t\|\nabla(X-Z)\|_{0}^{2} \leq & \eta(M) \Delta t\|\nabla(X-Z)\|_{0}^{2} \\
& +L \Delta t\|\nabla Z\|_{L^{\infty}}\|X-Z\|_{0}\|\nabla(X-Z)\|_{0} .
\end{aligned}
$$

After a kickback of $\|\nabla(X-Z)\|_{0}$, we obtain

$$
\begin{array}{r}
\|X-Z\|_{0}^{2}+(\lambda / 2) \Delta t\|\nabla(X-Z)\|_{0}^{2} \leq \eta(M) \Delta t\|\nabla(X-Z)\|_{0}^{2} \\
+\quad \frac{L^{2} \Delta t}{2 \lambda}\|\nabla Z\|_{L^{\infty}}^{2}\|X-Z\|_{0}^{2} .
\end{array}
$$

It follows from (4.12) and (4.13) that

$$
\Delta t\left\|\nabla E^{n}\right\|_{0}^{2} \leq C\left(\Delta t\left\|E^{n}\right\|_{0}^{2}+\left\|E^{n-1}\right\|_{0}^{2}+\Delta t e^{2}(\mathrm{HMM})\right) \leq C e^{2}(\mathrm{HMM}) .
$$

This, together with (4.8) and the inverse inequality, gives

$$
\Delta t^{1 / 2}\|\nabla Z\|_{L^{\infty}} \leq K_{1}+C H^{-1} \Delta t^{1 / 2}\left\|\nabla E^{n}\right\|_{0} \leq K_{1}+C H^{-1} e(\mathrm{HMM}) .
$$

Substituting the above inequality into (4.14), we get

$$
\|X-Z\|_{0}^{2}+(\lambda / 2) \Delta t\|\nabla(X-Z)\|_{0}^{2} \leq \eta(M) \Delta t\|\nabla(X-Z)\|_{0}^{2}+\left(L^{2} M^{2} / \lambda\right)\|X-Z\|_{0}^{2}
$$

with $M=K_{1}+C H^{-1} e(\mathrm{HMM})$. Using (4.10) and (4.11), we get $X=Z$, i.e. the HMM solution is locally unique.

Remark 4.4. Conditions (4.10) and (4.11) show that the HMM solution may not be unique if the estimating data procedure is not accurate enough. This is indeed the case even if the homogenized solution $U$ is unique. We refer to 3 for related discussion on the approximation of the quasilinear elliptic problems.

To simplify the presentation, we will show how to estimate $e(\mathrm{HMM})$ when (4.3) is changed slightly to

$$
\left\{\begin{aligned}
\partial_{t} v^{\varepsilon}-\nabla \cdot\left(a^{\varepsilon}\left(x, t, V\left(x_{K}\right)\right) \nabla v^{\varepsilon}\right) & =0 & & \text { in } \quad Q_{n}, \\
v^{\varepsilon} & =V & & \text { on } \quad \partial I_{\delta} \times T_{n}, \\
\left.v^{\varepsilon}\right|_{t=t_{n}} & =V, & &
\end{aligned}\right.
$$

and $A_{H}$ is changed to

$$
A_{H}\left(t_{n} ; V, W\right)=\sum_{K \in \mathcal{T}_{H}}|K| f_{Q_{n}} \nabla w^{\varepsilon} \cdot a^{\varepsilon}\left(x, t, V\left(x_{K}\right)\right) \nabla v^{\varepsilon} .
$$

Estimating $e(\mathrm{HMM})$ with cell problem (4.3) is more involved, and we will address it in a forthcoming paper.

Theorem 4.5. If we assume that $a^{\varepsilon}\left(x, t, u^{\varepsilon}\right)=a\left(x, x / \varepsilon, t, u^{\varepsilon}\right)$ with $a(x, y, t, p)$ periodic in $y$ with period $Y$, and the cell problem (4.15) is employed, then

$$
e(\mathrm{HMM}) \leq C\left(\delta+\left(\frac{\varepsilon}{\delta}\right)^{1 / 2}+\max _{1 \leq k \leq n}\left(\tau_{k}+\frac{\varepsilon}{\tau_{k}^{1 / 2}}\right)\right) .
$$

If $\left(\delta+(\varepsilon / \delta)^{1 / 2}+\tau_{n}+\varepsilon / \tau_{n}^{1 / 2}\right) / \Delta t^{1 / 2},\left(\delta+(\varepsilon / \delta)^{1 / 2}+\tau_{n}+\varepsilon / \tau_{n}^{1 / 2}\right) / H$, and $\Delta t / H$ are sufficiently small, then (4.10) and (4.11) hold. 
Proof. By the homogenization result in [4] and proceeding along the same line as (1.9), we may get (4.16). The only modification lies in the fact that $\mathcal{A}_{H}$ is not symmetric, therefore, the identity (3.13) is invalid, which actually accounts for the accuracy loss in (4.16).

To verify the validity of (4.10) and (4.11), we proceed in the same fashion of 17 , Theorem 5.5]. Define

$$
\mathcal{K}_{1}=\delta+\left(\frac{\varepsilon}{\delta}\right)^{1 / 2}+\tau_{n}+\frac{\varepsilon}{\tau_{n}^{1 / 2}}
$$

It follows from (4.16) that

$$
\left.L^{2} M^{2} \leq 2 L^{2}\left(K_{1}^{2}+C H^{-2} \mathcal{K}_{1}^{2}\right)=2 L^{2} C_{*}^{2}\left(\Delta t+H^{2}+(\Delta t / H)^{2}\right)+C L^{2} H^{-2} \mathcal{K}_{1}^{2}\right) .
$$

Therefore, there exists $\rho_{0}>0$ and $\rho_{1}>0$ such that if $\Delta t, H, \Delta t / H<\rho_{0}$ and $\mathcal{K}_{1} / H<\rho_{1}$, we get (4.10).

Next, proceeding in the same fashion as [17, Lemma 5.9], we may take $\eta(M)=$ $C\left(1+M \Delta t^{-1 / 2}\right) \mathcal{K}_{1}$. Invoking (4.16) once again, we obtain

$$
\begin{aligned}
\eta(M) & \leq C\left(1+K_{1} \Delta t^{-1 / 2}\right) \mathcal{K}_{1}+C H^{-1} \Delta t^{-1 / 2} \mathcal{K}_{1}^{2} \\
& \leq C\left(1+C_{*}\right) \mathcal{K}_{1}+C_{*}\left(H / \Delta t^{1 / 2}+\Delta t^{1 / 2} / H\right) \mathcal{K}_{1}+C H^{-1} \Delta t^{-1 / 2} \mathcal{K}_{1}^{2} .
\end{aligned}
$$

Therefore, there exists a constant $\rho_{2}$ such that if $\mathcal{K}_{1} / \Delta t^{1 / 2}<\rho_{2}$, we have $\eta(M)<$ $\lambda / 2$. Finally, let $\rho=\min \left(\rho_{1}, \rho_{2}\right)$; if $\mathcal{K}_{1} / \Delta t^{1 / 2}, \mathcal{K}_{1} / H<\rho$ and $\Delta t, H, \Delta t / H<\rho_{0}$, then (4.10) and (4.11) hold true.

Remark 4.6. A formal asymptotical expansion suggests that there is no oscillation in the temporal direction, and $u^{\varepsilon}$ in the coefficient $a^{\varepsilon}\left(x, x / \varepsilon, t, u^{\varepsilon}\right)$ serves as a parameter. Based on these special features of the problem, we may employ other types of cell problem and get a better estimate for $e(\mathrm{HMM})$. The details will be addressed elsewhere.

\section{Appendix A. ERror estimates for the LOCALly PERIODIC PARABOliC HOMOGENIZATION PROBLEMS}

The homogenization procedure for the parabolic problem is by now well understood; see [5, 6, 29] and the references therein. However, there are very few results concerning the error estimate for the difference between $u^{\varepsilon}$ and the homogenization solution $U$, or the difference between $u^{\varepsilon}$ and the first-order approximation $u_{1}^{\varepsilon}$ and the second-order approximation $u_{2}^{\varepsilon}$ (see (A.2) and (A.6) for the definitions). In this Appendix, we shall prove such error estimates for the locally periodic parabolic homogenization problem [6, 8].

As to the locally periodic parabolic homogenization problem, the homogenization matrix $\mathcal{A}$ is given by (3.6). We have the following regularity estimate for the solution of (1.2) (see [18]):

$$
\begin{aligned}
\|\nabla U\|_{L^{2}(\mathcal{Q})}+\left\|D^{2} U\right\|_{L^{2}(\mathcal{Q})} & \leq C\left(\|f\|_{L^{2}(\mathcal{Q})}+\left\|u_{0}\right\|_{1}\right), \\
\left\|\nabla \partial_{t} U\right\|_{L^{2}(\mathcal{Q})} & \leq C\left(\left\|\partial_{t} f\right\|_{L^{2}(\mathcal{Q})}+\left\|u_{0}\right\|_{2}\right) .
\end{aligned}
$$

Set

$$
u_{1}^{\varepsilon}:=U+\varepsilon \boldsymbol{\chi} \cdot \nabla U .
$$


A direct calculation yields

$$
\begin{aligned}
\left(a_{i j} \frac{\partial u_{1}^{\varepsilon}}{\partial x_{j}}\right)(x, x / \varepsilon, t) & =\left(\mathcal{A}_{i j} \frac{\partial U}{\partial x_{j}}\right)(x, t)+\mathcal{G}(x, x / \varepsilon, t) \nabla U \\
& +\varepsilon\left(a_{i j} \frac{\partial \chi^{k}}{\partial x_{j}}\right)(x, x / \varepsilon, t) \frac{\partial U}{\partial x_{k}}+\varepsilon\left(a_{i j} \chi^{k}\right)(x, x / \varepsilon, t) \frac{\partial^{2} U}{\partial x_{k} \partial x_{j}}
\end{aligned}
$$

where $\mathcal{G}=\left\{g_{i}^{j}\right\}_{i, j=1}^{d}$ is defined as

$$
g_{i}^{j}(x, y, t):=\left(a_{i j}+a_{i k} \frac{\partial \chi^{j}}{\partial y_{k}}\right)(x, y, t)-\mathcal{A}_{i j}(x, t) .
$$

Obviously,

$$
\int_{Y} g_{i}^{j}(x, y, t) \mathrm{d} y=0 \quad \text { and } \quad g_{i}^{j}(x, y, t) \text { is periodic in } y .
$$

Note that $\partial_{y_{i}} g_{i}^{j}(x, y, t)=0$ for $j=1, \ldots, d$; therefore, there exists a skew-symmetric matrix $\boldsymbol{\alpha}(x, y, t)=\left\{\alpha_{i j}^{k}(x, y, t)\right\}_{i, j, k=1}^{d}$ such that

$$
g_{i}^{j}(x, y, t)=\frac{\partial}{\partial y_{k}} \alpha_{i k}^{j}(x, y, t), \quad \int_{Y} \alpha_{i k}^{j}(x, y, t) \mathrm{d} y=0 .
$$

Thus, we obtain

$$
\begin{aligned}
g_{i}^{j}(x, x / \varepsilon, t) \frac{\partial U}{\partial x_{j}} & =\varepsilon \frac{\partial}{\partial x_{k}}\left(\alpha_{i k}^{j}(x, x / \varepsilon, t) \frac{\partial U}{\partial x_{j}}\right)-\varepsilon \alpha_{i k}^{j}(x, x / \varepsilon, t) \frac{\partial^{2} U}{\partial x_{k} \partial x_{j}} \\
& -\varepsilon \frac{\partial \alpha_{i k}^{j}}{\partial x_{j}}(x, x / \varepsilon, t) \frac{\partial U}{\partial x_{j}} .
\end{aligned}
$$

Let the corrector $\theta^{\varepsilon}$ be the solution of

$$
\left\{\begin{aligned}
\partial_{t} \theta^{\varepsilon}-\nabla \cdot\left(a(x, x / \varepsilon, t) \nabla \theta^{\varepsilon}\right) & =0 & & \text { in } \mathcal{Q}, \\
\theta^{\varepsilon} & =-\varepsilon \boldsymbol{\chi} \cdot \nabla U & & \text { on } \partial D \times(0, T), \\
\left.\theta^{\varepsilon}\right|_{t=0} & =-\left.\varepsilon \boldsymbol{\chi}\right|_{t=0} \cdot \nabla u_{0} & & \text { in } D .
\end{aligned}\right.
$$

Define

$$
u_{2}^{\varepsilon}:=u_{1}^{\varepsilon}+\theta^{\varepsilon} .
$$

We estimate $u^{\varepsilon}-u_{2}^{\varepsilon}$ in the following theorem.

Theorem A.1. Assume that $u_{0} \in H^{2}(D)$ and $f \in H^{1}\left(0, T ; L^{2}(D)\right)$. Then

$$
\begin{aligned}
\sup _{0<t \leq T}\left\|\left(u^{\varepsilon}-u_{2}^{\varepsilon}\right)(t)\right\|_{0} & +\left\|\nabla\left(u^{\varepsilon}-u_{2}^{\varepsilon}\right)\right\|_{L^{2}(\mathcal{Q})} \\
& \leq C \varepsilon\left(\left\|u_{0}\right\|_{2}+\|f\|_{L^{2}(\mathcal{Q})}+\left\|\partial_{t} f\right\|_{L^{2}(\mathcal{Q})}\right) .
\end{aligned}
$$

Proof. For any $\phi \in \mathcal{C}\left(0, T ; L^{2}(D)\right) \cap L^{2}\left(0, T ; H_{0}^{1}(D)\right)$ with $\phi(x, 0)=0$, we write the weak form of (1.2) and (A.5) as

$$
\int_{D}\left(\phi \partial_{s} U+\nabla \phi \cdot \mathcal{A} \nabla U\right) \mathrm{d} x=\int_{D} f \phi \mathrm{d} x \quad \text { and } \quad \int_{D}\left(\phi \partial_{s} \theta^{\varepsilon}+\nabla \phi \cdot a^{\varepsilon} \nabla \theta^{\varepsilon}\right) \mathrm{d} x=0 .
$$


Invoking (A.3) and the above equations, we obtain

$$
\begin{aligned}
& \int_{D} \phi \partial_{s}\left(u^{\varepsilon}-u_{2}^{\varepsilon}\right) \mathrm{d} x+\int_{D} \nabla \phi \cdot a^{\varepsilon} \nabla\left(u^{\varepsilon}-u_{2}^{\varepsilon}\right) \mathrm{d} x \\
& =-\varepsilon \int_{D} \partial_{s}(\chi \cdot \nabla U) \phi \mathrm{d} x-\int_{D} \nabla \phi \cdot \mathcal{G} \nabla U \mathrm{~d} x-\varepsilon \int_{D} \nabla \phi \cdot a^{\varepsilon} \nabla(\chi \cdot \nabla U) \mathrm{d} x .
\end{aligned}
$$

In view of (A.4) and the fact that $\boldsymbol{\alpha}$ is a skew-symmetric matrix, we get

$$
\int_{D} \nabla \phi \cdot \mathcal{G} \nabla U \mathrm{~d} x=-\varepsilon \int_{D}\left(\nabla \phi \cdot \boldsymbol{\alpha}: D^{2} U+\nabla \phi \cdot(\nabla \cdot \boldsymbol{\alpha}) \nabla U\right) \mathrm{d} x .
$$

Substituting the above identity into (A.8), we get

$$
\begin{aligned}
& \int_{D} \partial_{s}\left(u^{\varepsilon}-u_{2}^{\varepsilon}\right) \phi \mathrm{d} x+\int_{D} \nabla \phi \cdot a^{\varepsilon} \nabla\left(u^{\varepsilon}-u_{2}^{\varepsilon}\right) \mathrm{d} x \\
& =-\varepsilon \int_{D} \partial_{s}(\boldsymbol{\chi} \cdot \nabla U) \phi \mathrm{d} x-\varepsilon \int_{D} \nabla \phi \cdot a^{\varepsilon} \nabla(\boldsymbol{\chi} \cdot \nabla U) \mathrm{d} x \\
& \quad+\varepsilon \int_{D}\left(\nabla \phi \cdot \boldsymbol{\alpha}: D^{2} U+\nabla \phi \cdot(\nabla \cdot \boldsymbol{\alpha}) \nabla U\right) \mathrm{d} x .
\end{aligned}
$$

Taking $\phi=u^{\varepsilon}-u_{2}^{\varepsilon}$ in the above identity since $\left(u^{\varepsilon}-u_{2}^{\varepsilon}\right) \in H_{0}^{1}(D)$ and $\left.\left(u^{\varepsilon}-u_{2}^{\varepsilon}\right)\right|_{t=0}=$ 0 , integrating from 0 to $t$ for any $0<t \leq T$, we obtain

$$
\left\|\left(u^{\varepsilon}-u_{2}^{\varepsilon}\right)(x, t)\right\|_{0}+\left(\int_{0}^{t}\left\|\nabla\left(u^{\varepsilon}-u_{2}^{\varepsilon}\right)\right\|_{0}^{2} \mathrm{~d} s\right)^{1 / 2} \leq C \varepsilon\left(\int_{0}^{t}\left(\left\|\partial_{s} U\right\|_{1}^{2}+\|U\|_{2}^{2}\right) \mathrm{d} s\right)^{1 / 2} .
$$

A combination of the above inequality and the regularity estimate (A.1) gives A.7).

In what follows, we turn to the estimates for the corrector and the first order approximation $u_{1}^{\varepsilon}$. No error estimates for the correctors are available to the best of the author's knowledge.

Theorem A.2. Assume that $u_{0} \in H^{2}(D)$ and $f, \partial_{t} f \in L^{2}(Q)$. Then

$$
\begin{aligned}
& \sup _{0<t \leq T}\left\|\left(u^{\varepsilon}-u_{1}^{\varepsilon}\right)(t)\right\|_{0}+\left\|\nabla\left(u^{\varepsilon}-u_{1}^{\varepsilon}\right)\right\|_{L^{2}(\mathcal{Q})} \\
& \leq C \sqrt{\varepsilon}\left(\left\|u_{0}\right\|_{2}+\|f\|_{L^{2}(\mathcal{Q})}+\left\|\partial_{t} f\right\|_{L^{2}(\mathcal{Q})}\right)
\end{aligned}
$$

and

$$
\sup _{0<t \leq T}\left\|\left(u^{\varepsilon}-U\right)(t)\right\|_{0} \leq C \sqrt{\varepsilon}\left(\left\|u_{0}\right\|_{2}+\|f\|_{L^{2}(\mathcal{Q})}+\left\|\partial_{t} f\right\|_{L^{2}(\mathcal{Q})}\right) .
$$

Proof. Define $\psi^{\varepsilon} \in \mathcal{C}_{0}^{\infty}(D)$, which equals 1 in $D / D_{2 \varepsilon}$ and equals 0 in $D_{\varepsilon}$, where

$$
D_{\varepsilon}:=\{x \in D \mid \operatorname{dist}(x, \partial D) \leq \varepsilon\} .
$$

Obviously, $\left|\nabla \psi^{\varepsilon}\right| \leq C / \varepsilon$. 
Define $w^{\varepsilon}:=U+\varepsilon \psi^{\varepsilon} \boldsymbol{\chi} \cdot \nabla U$; obviously, $w^{\varepsilon}(x, t) \in H_{0}^{1}(D)$ for a.e.t $\in(0, T]$. A direct calculation gives

$$
\begin{aligned}
\sup _{0<t \leq T} & \left\|\left(u_{1}^{\varepsilon}-w^{\varepsilon}\right)(t)\right\|_{L^{2}(D)}+\left\|\nabla\left(u_{1}^{\varepsilon}-w^{\varepsilon}\right)\right\|_{L^{2}(\mathcal{Q})} \\
\leq & C C \sqrt{\varepsilon}\left(\left\|u_{0}\right\|_{1}+\|\nabla U\|_{L^{2}(\mathcal{Q})}+\left\|\nabla \partial_{t} U\right\|_{L^{2}(\mathcal{Q})}+\left\|D^{2} U\right\|_{L^{2}(\mathcal{Q})}\right) .
\end{aligned}
$$

It remains to bound $u^{\varepsilon}-w^{\varepsilon}$. As that in the proof of (A.7), we have for any $\phi \in \mathcal{C}\left(0, T ; L^{2}(D)\right) \cap L^{2}\left(0, T ; H_{0}^{1}(D)\right)$,

$$
\begin{aligned}
\int_{D} \partial_{s}\left(u^{\varepsilon}-w^{\varepsilon}\right) \phi \mathrm{d} x+\int_{D} \nabla \phi \cdot a^{\varepsilon} \nabla\left(u^{\varepsilon}-w^{\varepsilon}\right) \mathrm{d} x \\
=-\varepsilon \int_{D} \partial_{s}(\chi \cdot \nabla U) \phi \psi^{\varepsilon} \mathrm{d} x+\varepsilon \int_{D} \nabla \phi \cdot a^{\varepsilon} \nabla(\boldsymbol{\chi} \cdot \nabla U) \mathrm{d} x \\
\quad-\varepsilon \int_{D} \nabla \phi \cdot a^{\varepsilon}\left(\boldsymbol{\chi} \cdot D^{2} U\right) \psi^{\varepsilon} \mathrm{d} x-\int_{D} \boldsymbol{r}^{\varepsilon} \cdot \nabla \phi \mathrm{d} x,
\end{aligned}
$$

where $\boldsymbol{r}^{\varepsilon}$ is defined by

$$
\boldsymbol{r}^{\varepsilon}:=\nabla U \cdot a^{\varepsilon} \nabla_{y} \chi\left(\psi^{\varepsilon}-1\right)+\varepsilon \nabla \psi^{\varepsilon} \cdot a^{\varepsilon}(\chi \nabla U) .
$$

The terms except the last one in the right-hand side of of the above expansion can be easily bounded by $C \varepsilon\left(\left|\partial_{s} U\right|_{1}+|U|_{1}+|U|_{2}\right)\|\nabla \phi\|_{0}$.

By virtue of [21, Lemma 2.5], we get

$$
|U|_{1, D_{2 \varepsilon}} \leq C \sqrt{\varepsilon}\left(|U|_{1}+|U|_{2}\right) .
$$

We thus bound $\boldsymbol{r}^{\varepsilon}$ as

$$
\left\|\boldsymbol{r}^{\varepsilon}\right\|_{0} \leq C|U|_{1, D_{2 \varepsilon}} \leq C \sqrt{\varepsilon}\left(|U|_{1}+|U|_{2}\right) .
$$

Therefore, we get

$$
\int_{D} \partial_{s}\left(u^{\varepsilon}-w^{\varepsilon}\right) \phi \mathrm{d} x+\int_{D} \nabla \phi \cdot a^{\varepsilon} \nabla\left(u^{\varepsilon}-w^{\varepsilon}\right) \mathrm{d} x \leq C \sqrt{\varepsilon}\left(\left|\partial_{s} U\right|_{1}+|U|_{1}+|U|_{2}\right)\|\nabla \phi\|_{0},
$$

and let $\phi=u^{\varepsilon}-w^{\varepsilon}$. Integrating the above inequality from 0 to $t$, we obtain

$$
\begin{aligned}
\left\|\left(u^{\varepsilon}-w^{\varepsilon}\right)(t)\right\|_{0}^{2}+\int_{0}^{t}\left\|\nabla\left(u^{\varepsilon}-w^{\varepsilon}\right)\right\|_{0}^{2} \leq & \left\|\left(u^{\varepsilon}-w^{\varepsilon}\right)(x, 0)\right\|_{0}^{2} \\
& +C \varepsilon \int_{0}^{t}\left(\left\|\partial_{s} U\right\|_{0}^{2}+\|\nabla U\|_{0}^{2}+\left\|D^{2} U\right\|_{0}^{2}\right) \mathrm{d} s .
\end{aligned}
$$

Using $\left\|\left(u^{\varepsilon}-w^{\varepsilon}\right)(x, 0)\right\|_{0} \leq C \varepsilon\left\|u_{0}\right\|_{1}$, we get

$$
\begin{aligned}
\max _{0<t \leq T}\left\|\left(u^{\varepsilon}-w^{\varepsilon}\right)(t)\right\|_{0} & +\left\|\nabla\left(u^{\varepsilon}-w^{\varepsilon}\right)\right\|_{L^{2}(\mathcal{Q})} \\
& \leq C \sqrt{\varepsilon}\left(\left\|u_{0}\right\|_{1}+\left\|\nabla \partial_{t} U\right\|_{L^{2}(\mathcal{Q})}+\left\|D^{2} U\right\|_{L^{2}(\mathcal{Q})}\right) .
\end{aligned}
$$

This inequality together with (A.12) and the regularity estimate (A.1) give the desired estimate (A.10). The estimate (A.11) follows from (A.7) and (A.10).

If $U$ is smoother, then we may improve (A.11) from $\mathcal{O}(\sqrt{\varepsilon})$ to $\mathcal{O}(\varepsilon)$. 
Corollary A.3. If $\nabla U \in L^{\infty}(\mathcal{Q})$, then we have

$$
\sup _{0<t \leq T}\left\|\left(u^{\varepsilon}-U\right)(t)\right\|_{0} \leq C \varepsilon\left(\left\|u_{0}\right\|_{2}+\|f\|_{L^{2}(\mathcal{Q})}+\left\|\partial_{t} f\right\|_{L^{2}(\mathcal{Q})}+\|\nabla U\|_{L^{\infty}(\mathcal{Q})}\right) .
$$

Proof. By maximum principle [20], we have

$$
\max _{(x, t) \in \mathcal{Q}}\left|\theta^{\varepsilon}(x, t)\right| \leq C \varepsilon \max _{(x, t) \in \mathcal{Q}}|\nabla U(x, t)|,
$$

which together with (A.7) gives

$$
\begin{aligned}
\sup _{0<t \leq T}\left\|\left(u^{\varepsilon}-U\right)(t)\right\|_{0} \leq & \sup _{0<t \leq T}\left\|\left(u^{\varepsilon}-u_{2}^{\varepsilon}\right)(t)\right\|_{0} \\
& +\varepsilon \sup _{0<t \leq T}\|(\chi \cdot \nabla U)(t)\|_{0}+\sup _{0<t \leq T}\left\|\theta^{\varepsilon}(\cdot, t)\right\|_{0} \\
\leq & C \varepsilon\left(\left\|u_{0}\right\|_{2}+\|f\|_{L^{2}(\mathcal{Q})}+\left\|\partial_{t} f\right\|_{L^{2}(\mathcal{Q})}\right) \\
& +C \varepsilon \max _{0<t \leq T}\|\nabla U(\cdot, t)\|_{0}+C \varepsilon\|\nabla U\|_{L^{\infty}(\mathcal{Q})} \\
\leq & C \varepsilon\left(\left\|u_{0}\right\|_{2}+\|f\|_{L^{2}(\mathcal{Q})}+\left\|\partial_{t} f\right\|_{L^{2}(\mathcal{Q})}+\|\nabla U\|_{L^{\infty}(\mathcal{Q})}\right) .
\end{aligned}
$$

This gives (A.13).

Note that (A.14) also holds true for the case when $a^{\varepsilon}=a\left(x, x / \varepsilon, t, t / \varepsilon^{2}\right)$. Therefore, we may proceed as in Lemma 3.3 to obtain the following estimate (A.15) for the corrector. But we cannot obtain (A.10) since we cannot obtain (A.7) by the method herein.

Corollary A.4. For $a^{\varepsilon}=a\left(x, x / \varepsilon, t, t / \varepsilon^{2}\right)$ with $a(\cdot, y, \cdot s)$ periodic in $y$ and $s$, respectively, with periods $Y$ and 1 , if $\nabla U \in L^{\infty}(\mathcal{Q})$, then we have

$$
\left\|\nabla \theta^{\varepsilon}\right\|_{L^{2}(\mathcal{Q})} \leq C \sqrt{\varepsilon}\left(\left\|u_{0}\right\|_{2}+\|f\|_{L^{2}(\mathcal{Q})}+\left\|\partial_{t} f\right\|_{L^{2}(\mathcal{Q})}+\|\nabla U\|_{L^{\infty}(\mathcal{Q})}\right) .
$$

Remark A.5. In case of the one-dimensional problem, the following error estimates are stated in [5, p. 43, Theorem 1]:

$$
\left\|\nabla\left(u^{\varepsilon}-u_{2}^{\varepsilon}\right)\right\|_{L^{2}(\mathcal{Q})} \leq C(T) \varepsilon, \quad\left\|\nabla\left(u^{\varepsilon}-u_{1}^{\varepsilon}\right)\right\|_{L^{2}(\mathcal{Q})} \leq C(T) \varepsilon .
$$

It is not surprising that the error estimate for the first-order approximation is $\mathcal{O}(\varepsilon)$, since there is no boundary layer for one-dimensional problem.

\section{ACKNOWLEDGMENTS}

We thank the anonymous referees for the improvements of this paper. In particular Corollaries A.3 and A.4.

\section{REFERENCES}

1. A. Abdulle and W. E, Finite difference heterogeneous multi-scale method for homogenization problems, J. Comput. Phys. 191 (2003), 18-39. MR2008485 (2004i:65071)

2. G. Alexopoulos, An application of homogenization theory to harmonic analysis: Harnack inequalities and Reisz transforms on Lie groups of polynomial growth, Canad. J. Math. 44 (1992), 691-727. MR 1178564 (93j:22006)

3. N. André and M. Chipot, Uniqueness and nonuniqueness for the approximation of quasiliear elliptic equations, SIAM J. Numer. Anal. 33 (1996), 1981-1994. MR1411859 (98k:65064)

4. M. Artola and G. Duvaut, Un résultat d'homogénéisation pour une classe de problémes de diffusion non stationnaires, Ann. Fac. Sci. Toulouse. (5) IV (1982), 1-28. MR0673637 $(84 \mathrm{j}: 35020)$

5. N. Bakhvalov and G. Panasenko, Homogenisation: Averaging Processes in Periodic Media, Mathematical Problems in the Mechanics of Materials, Kluwer Academic Publishers, 1989. MR.1112788 (92d:73002) 
6. A. Bensoussan, J. L. Lions and G. C. Papanicolaou, Asymptotic Analysis for Periodic Structures, North-Holland, Amsterdam, 1978. MR0503330 (82h:35001)

7. L. Boccardo and F. Murat, Homogénéisation de problémes quasi-linéaires, Publ. IRMA, Lille. 3 (1981), no. 7, 13-51. MR0766874 (86a:35022)

8. S. Brahim-Otsmane, G. A. Francfort and F. Murat, Correctors for the homogenization of the wave and heat equations, J. Math. Pures Appl. 71 (1992), 197-231. MR1172450 (93d:35012)

9. S. Q. Chen, W. E and C-W. Shu, The heterogeneous multiscale method based on the discontinuous Galerkin method for hyperbolic and parabolic problems, Multiscale Model. Simul. 3 (2005), 871-894. MR2164241 (2006h:65142)

10. P. G. Ciarlet, The Finite Element Method for the Elliptic Problems, North-Holland, Amsterdam, 1978. MR0520174 (58:25001)

11. F. Colombini and S. Spagnolo, Sur la convergence de solutions d'équations paraboliques, J. Math. Pures Appl. 56 (1977), 263-305. MR0603300 (58:29248)

12. A. Dall'aglio and F. Murat, A corrector result for H-converging parabolic problems with time-dependent coefficients, Ann. Scuola Norm. Sup. Pisa C1. Sci. XXV (1997), 329-373. MR.1655521 (99m:35023)

13. J. Douglas and T. Dupont, Galerkin methods for parabolic equations, SIAM J. Numer. Anal. 7 (1970), 575-626. MR0277126 (43:2863)

14. W. E, Analysis of the heterogeneous multiscale method for ordinary differential equations, Commun. Math. Sci. 1 (2003), 423-436. MR2069938 (2005f:65082)

15. W. E and B. Engquist, The heterogeneous multi-scale methods, Commun. Math. Sci. 1 (2003), 87-132. MR.1979846 (2004b:35019)

16. W. E and B. Engquist, Multiscale modeling and computation, Notices Amer. Math. Soc. 50 (2003), 1062-1070. MR2002752 (2004m:65163)

17. W. E, P. B. Ming and P. W. Zhang, Analysis of the heterogeneous multiscale method for elliptic homogenization problems, J. Amer. Math. Soc. 18 (2005), 121-156. MR2114818(2005k:65246)

18. L. C. Evans, Partial Differential Equations, American Mathmatical Society, Providence, Rhode Island: AMS, 1998. MR1625845 (99e:35001)

19. J. Garcia-Azorero, C. E. Gutierrez and I. Peral, Homogenization of quasilinear parabolic equations in periodic media, Comm. Partial Differential Equations. 28 (2003), 1887-1910. MR:2015406 (2004i:35028)

20. O. A. Ladyzhenskaya, V. A. Solonnikov and N. N. Ural'tseva, Linear and Quasilinear Equations of Parabolic Type, Translations of Mathematics Monographys, Vol. 25, Providence, Rhode Island: AMS, 1968. MR.1195131 (93k:35025)

21. O. A. Oleinik, A. S. Shamaev and G. A. Yosifian, Mathematical Problems in Elasticity and Homogenization, North-Holland, Amsterdam, 1992. MR1195131 (93k:35025)

22. S. Spagnolo, Sul limite delle soluzioni di problemi di Cauchy relativi all'equazione del calore, Ann. Scuola Norm. Sup. Pisa (3) 21 (1967), 657-699. MR0225015 (37:612)

23. S. Spagnolo, Sulla convergenza di soluzioni di equationi paraboliche ed ellittiche, Ann. Scuola Norm. Sup. Pisa (3) 22 (1968), 571-597. MR0240443 (39:1791)

24. S. Spagnolo, Convergence of parabolic equations, Boll. Un. Mat. Ital. B (5) 14 (1977), 547-568. MR $0460889(57: 880)$

25. L. Tartar, H-Convergence, Course Peccot, Collége de France, March 1977. Partially written by F. Murat. Séminaire d'Analyse Fonctionnelle et Numérique de l'Université d'Alger, 1977-1978. English Translation: F. Murat and L. Tartar: H-Convergence, in Topics in the Mathematical Modeling of Composite Materials, A. Cherkaev and R. Kohn, eds., Birkhäuser, Boston, 1997, pp. 21-43. MR1493039

26. V. Thomée, Galerkin Finite Element Methods for Parabolic Problems, Springer-Verlag, Berlin, Heidelberg, 1997. MR1479170 (98m:65007)

27. V. V. Zhikov, S. M. Kozlov and O. A. Oleinik, G-convergence of parabolic operators, Uspekhi Mat. Nauk 36: 1 (1981), 11-58. English Translation: Russ. Math. Surv. 36 (1981), 9-60. MR.0608940 (83a:35055)

28. V. V. Zhikov, S. M. Kozlov and O. A. Oleinik, Averaging of parabolic operators, Trudy Mosk. Mat. O.-va 45 (1982), 182-236. English Translation: Trans. Moscow Math. Soc. 45 (1984), 189-241. MR0704631 (85k:35024)

29. V. V. Zhikov, S. M. Kozlov and O. A. Oleinik, Homogenization of Differential Operators and Integral Functionals, Springer-Verlag, Berlin, Heidelberg, 1994. MR.1318242 (96h:35003a) and 1329546 (96h:35003b) 
LSEC, Institute of Computational Mathematics and Scientific/Engineering Computing, AMSS, Chinese Academy of Sciences, No. 55 Zhong-Guan-Cun East Road, Beijing, 100080, People's Republic of China

E-mail address: mpb@lsec.cc.ac.cn

Lmam and School of Mathematical Sciences, Peking University, Beijing, 100871, People's Republic of China

E-mail address: pzhang@pku.edu.cn 